\title{
Le aree prioritarie per la biodiversità della provincia di Novara Una proposta multidisciplinare
}

\author{
Giuseppe Bogliani ${ }^{1 *}$, Fabio Casale ${ }^{2}$, Claudio Celada ${ }^{2}$, Luciano Crua ${ }^{3}$, \\ Romina Di Paolo ${ }^{3}$, Massimiliano Ferrarato ${ }^{3}$, Nicola Gilio' ${ }^{2}$, Federica Luoni ${ }^{2}$, \\ Matteo Massara ${ }^{4}$, Tiziana Masuzzo ${ }^{5}$, Massimo Soldarini ${ }^{2}$, Davide Vietti ${ }^{3}$
}

\begin{abstract}
Priority areas for biodiversity conservation in the province of Novara, Italy. A multidisciplinary approach.

This research has identified the Priority Areas for Biodiversity Conservation for the Province of Novara, Piedmont Region, Italy, as a baseline for planning the Provincial Ecological Network. 26 expert naturalists participated in this operation by sharing their personal knowledge about the territory of the province of Novara for the following six themes: (a) Flora and vegetation, (b) Invertebrates, (c) Amphibians and Reptiles, (d) Birds, (e) Mammals, (f) Aquatic Ecosystems and Fishes. Experts were asked to identify the most representative focal themes for their topic and assign a naturalistic value to different portions of the provincial territory at the top for the theme. The methodology adopted, based on gathering direct information from top experts in the area, has been integrated, for some little explored portions of the territory, with GIS modeling based on land use and human pressure databases. This allowed mapping of the most important areas for biodiversity conservation. The gap analysis of Priority Areas with Protected Areas (Regional Parks, Nature Reserves, Natura 2000 sites such as Special Areas of Conservation and Special Protection Areas) allowed us to evaluate the effectiveness of these latter areas for the conservation of biodiversity. Priority Areas were actually located almost totally but not exclusively in Protected Areas. However, the Protected Areas system of the Novara province did not take into account significant portions of territory with high-value biodiversity that, thanks to this research, are now identified.
\end{abstract}

Key-words: Italy, Piedmont, Novara, biodiversity, protected areas, gap analysis, conservation priority.

Riassunto - Con questa ricerca sono state individuate le Aree prioritarie per la conservazione della biodiversità della provincia di Novara quale operazione di partenza al fine di disegnare, in una fase successiva, la Rete Ecologica Provinciale su basi naturalistiche. A questa operazione hanno partecipato 26 naturalisti esperti, ossia dotati di compe-

\footnotetext{
' Dipartimento di Scienze della terra e dell'ambiente, Università degli Studi di Pavia, Via Adolfo Ferrata 9, 27100 Pavia.

${ }^{2}$ Lipu - BirdLife Italia, via Udine 3/A, 43122 Parma.

${ }^{3}$ ARPA Piemonte - Agenzia regionale per la protezione ambientale, Settore Ambiente e Natura, Via Pio VII, 9, 10135 Torino.

${ }^{4}$ Regione Piemonte, Settore Biodiversità e aree naturali, Via Principe Amedeo, 17, 10123 Torino.

${ }^{5}$ Provincia di Novara, Settore Urbanistica e Trasporti, Via Greppi, 7, 28100 Novara
}

\section{* Corresponding author: giuseppe.bogliani@unipv.it}

Received: 10 December 2016

Accepted for publication: 12 May 2017 tenze personali pregresse e di adeguata conoscenza del territorio della provincia di Novara, per i seguenti 6 temi focali: (a) Flora e vegetazione, (b) Invertebrati, (c) Anfibi e Rettili, (d) Uccelli, (e) Mammiferi, (f) Ecosistemi acquatici e pesci. Agli esperti è stato chiesto di individuare i temi focali più rappresentativi per la loro disciplina e di assegnare in modo comparativo un valore naturalistico di aree diverse del territorio provinciale. La metodologia adottata, basata sull'ottenimento delle informazioni dirette da parte dei maggiori esperti presenti sul territorio, è stata integrata per porzioni del territorio poco esplorato con un'analisi in ambiente GIS sulla base di database dell'uso del suolo e delle pressioni antropiche. Questo ha permesso di cartografare le aree più importanti per la conservazione della biodiversità. La gap analysis delle Aree prioritarie con le Aree protette e con altre categorie di tutela del territorio già presenti (parchi e riserve naturali, SIC, ZPS), ha permesso di valutare l'efficacia di queste seconde per la conservazione della biodiversità. Le Aree prioritarie sono state effettivamente localizzate quasi totalmente ma non esclusivamente in Aree protette. Tuttavia, l'insieme delle Aree protette del territorio novarese escludeva porzioni significative di territori di elevato valore per la biodiversità, ora meglio definiti con questa ricerca.

Parole chiave: Italia, Piemonte, Novara, biodiversità, aree protette, gap analysis, priorità nella conservazione.

\section{INTRODUZIONE}

L'individuazione delle aree più importanti per la biodiversità sulla base di una pluralità di indicatori è stata strumentale nelle scelte di conservazione a livello internazionale, nazionale, regionale e locale, soprattutto allo scopo di individuare le aree da sottoporre a particolari regimi di tutela e pratiche di gestione, come parchi e riserve naturali (Knight, 2008; Moilanen et al., 2008). L'esigenza di definire delle priorità nella conservazione delle aree è ancor più urgente in questo periodo caratterizzato da cambiamenti significativi e rapidi nella distribuzione delle popolazioni di specie vegetali e animali, in conseguenza dell'espansione delle aree antropizzate e dei cambiamenti globali (Li et al., 2013; Loyola et al., 2013). Nel passato, la rete delle aree protette è stata definita sulla base delle conoscenze naturalistiche esistenti al momento della loro individuazione, spesso parziali. In Italia le aree protette sono state istituite prevalentemente dopo l'entrata in vigore della riforma delle regioni a statuto ordinario nel 1970. In Piemonte, in particolare, la designazione delle aree protette ha preso il via nel 1975 ed è stata integrata da successivi provvedimenti legislativi, attraverso la Legge regionale n. 19 del 29 giugno 2009. I criteri utilizzati nel 1975 erano stati essenzialmente i seguenti: 
1 - Il territorio è compreso nell'elenco dei biotopi di interesse botanico della Società Botanica Italiana (Gruppo Di Lavoro Per La Conservazione Della Natura Della Società Botanica Italiana, 1971, 1979).

2 - Il territorio ospita elementi faunistici e floristici di spicco (garzaie, ecc.).

3 - Esistono complessi monumentali religiosi diffusi in una matrice naturalistica, chiamati Sacri Monti.

Quindi, la designazione della Aree Protette piemontesi risale ad alcuni decenni fa; nel frattempo gli studi naturalistici sono proseguiti e sono state individuate nuove aree di valore naturalistico e sono state meglio caratterizzate quelle già note. Inoltre, nel frattempo il gruppo di potenziali indicatori di biodiversità è aumentato considerevolmente, grazie all'intensificarsi delle ricerche. È quindi possibile che le aree protette esistenti, anche nei casi nei quali lo stato di conservazione non è nel frattempo peggiorato, non siano sufficienti a garantire la conservazione delle popolazioni e delle cenosi meritevoli di tutela in base al livello di minaccia. Inoltre, secondo l'analisi sintetica soggettiva di Framarin $(1981,1982)$, non chiaramente descritta nei particolari e pertanto non replicabile, sin dall'inizio del processo di selezione delle aree protette in Piemonte, non tutti i biotopi maggiormente rilevanti erano inclusi.

L'uso di taxa, habitat o temi focali indicatori è diffuso nei processi di pianificazione territoriale e di delimitazione delle aree protette. Questo approccio è talvolta l'unico praticabile, in quanto il livello delle conoscenze non è sufficientemente dettagliato e non consente di prendere in considerazione tutte le possibili componenti (popolazioni, specie, comunità, ecosistemi). I pareri sull'utilità di questo approccio sono discordanti e a fronte di valutazioni positive (p.e. Sætersdal et al., 1993), sono state formulate critiche che tuttavia tendono ad attenuarsi quando si procede con un numero relativamente elevato di indicatori (p.e. Howard et al., 1998). In Piemonte, nonostante l'enorme incremento del livello di conoscenza naturalistica del territorio degli scorsi decenni, alcune aree risultano tutt'ora poco esplorate. In questi casi, l'uso di indicatori oggettivi, per i quali non sono disponibili dati, può essere insufficiente per individuare le aree di maggior pregio naturalistico.

In altre circostanze analoghe, al fine di identificare e delimitare le aree più importanti per la conservazione della biodiversità, in carenza di dati, ci si è orientati recentemente, con frequenza crescente, all'uso di modelli matematici. Questi disegnano mappe presuntive di distribuzione e/o di idoneità in base alle informazioni disponibili sulla distribuzione e qualche volta sull'idoneità ambientale per un numero limitato di indicatori, popolazioni o taxa; lo fanno attraverso algoritmi appropriati e grazie alla disponibilità di basi di dati ambientali e geografiche (Franklin, 2009). Tali modelli permettono di integrare e sintetizzare le relazioni specie-ambiente e rappresentano un valido strumento di supporto alle indagini conoscitive e ai progetti di conservazione e gestione territoriale (Brambilla et al., 2009; Milanesi et al., 2015; Balestrieri et al., 2016). Essi restituiscono una cartografia delle aree in grado di rappresentare diversi livelli di qualità di habitat per ogni specie. La sovrapposizione dei poligoni che delimitano i valori assunti dagli indicatori di singole specie consente di individuare le aree nelle quali si riscontrano i valori sommati maggiori. Un esempio ben conosciuto in Italia è quello della REN-Rete Ecologica Nazionale (Boitani et al., 2002).

Nel caso di specie selvatiche, talora molto elusive e difficilmente censibili, l'approccio modellistico sopra descritto rappresenta spesso, sulla base di una valutazione costi/benefici, l'unica possibilità per definire a priori l'idoneità di un ambiente a sostenere una popolazione animale. L'affidabilità dei modelli è però condizionata da diversi fattori quali la disponibilità, la precisione e l'omogeneità dei dati per le diverse aree di studio. Questo approccio può rivelarsi idoneo a descrivere le distribuzioni attese quando le basi di dati ambientali che si utilizzano per generare i modelli di distribuzione dei taxa utilizzati sono di buona qualità, aggiornate e idonee a spiegare le relazioni con le variabili influenzanti la distribuzione, l'abbondanza e la fitness degli organismi considerati. Tuttavia, nessuna base di dati finora conosciuta è in grado di contenere tutte le informazioni possibili.

Fra le informazioni difficili da ottenere ci sono quelle relative alla storia delle diverse porzioni di territorio, soprattutto in relazione alle modificazioni indotte dall'attività umana; questa può essere ancora in atto e quindi misurata e introdotta nei modelli fra le variabili indipendenti; oppure è terminata da tempo ma dopo aver lasciato conseguenze ambientali spesso non più misurabili sul campo ma in grado di modificare $\mathrm{i}$ valori di idoneità per le specie e gli habitat. L'approccio modellistico diventa indispensabile per individuare e prevedere gli effetti di processi ecologici ai quali non è dato di assistere localmente, quali gli eventi di dispersione degli organismi da un'area idonea a un'altra. In questi casi, la necessità di individuare i fattori riconoscibili sul terreno e nelle basi dati ambientali e di correlarli a probabili comportamenti ed eventi (l'organismo attraversa l'area, vi si sofferma, o la evita) o esiti (l'organismo ha probabilità variabile di transitare nell'area con esito non letale), ha portato all'elaborazione di diversi approcci modellistici (p.e. Adriaensen et al., 2004) applicabili a diverse realtà territoriali.

\section{Lo stato delle conoscenze per il territorio della provincia di Novara}

Nella provincia di Novara, all'inizio del progetto "Novara in Rete", il grado di conoscenza su distribuzione, abbondanza e vitalità di alcuni taxa o temi focali relativi alla biodiversità era talvolta buono e, comunque, sufficiente a operare con una pluralità di indicatori su alcune porzioni del territorio provinciale. Ciononostante, rimanevano delle porzioni di territorio relativamente meno conosciute.

Per alcuni gruppi di organismi il lavoro di individuazione delle aree di maggior rilevanza era stato condotto dai ricercatori, nel recente passato, secondo standard rigorosi e ripetibili. Questo era avvenuto soprattutto per le componenti botanica e ornitologica. Per esempio, Selvaggi et al. (2010) avevano individuato le IPA- 
Important Plant Areas del Piemonte e per la provincia di Novara hanno indicato: 1) PIEM 4 Lago d'Orta, T. Pescone e torbiera Valle Scoccia; 2) PIEM 5 Lagoni di Mercurago, Canneti di Dormelletto e Bosco Solivo. Tuttavia, Selvaggi et al. (2010) avevano rilevato fra le criticità del lavoro le lacune di conoscenze in alcune parti del Novarese.

Per gli Uccelli, classe di Vertebrati particolarmente studiata, in provincia di Novara erano state individuate da Brunner et al. (2002) le seguenti IBA-Important Bird Areas: 1) Garzaie del Novarese; 2) Garzaie del Sesia (in condivisione con la provincia di Vercelli); 3) Fiume Ticino (in condivisione con la regione Lombardia). La componente ornitologica, inoltre, era stata studiata in buon dettaglio in diversi altri suoi aspetti; la qualità e quantità di dati e la densità dei rilevatori che avevano collaborato alla raccolta delle informazioni consentivano di delineare un buon quadro di dettaglio (Fasola et al., 1981; Mingozzi et al., 1988; Cucco et al., 1996; Bordignon, 2004; Aimassi \& Reteuna, 2007). Nell'ambito del progetto "Novara in Rete" sotto descritto, inoltre, l'indagine ornitologica è stata ulteriormente approfondita e, soprattutto, indirizzata a colmare le lacune conoscitive (Casale et al., 2017).

Per altri taxa o temi focali non erano state formalizzate liste analoghe alle due precedenti. Tuttavia, il livello delle conoscenze disponibili aveva consentito di individuare biotopi o aree rilevanti sulla base della presenza di specie rare e minacciate o di comunità ricche e diversificate. È il caso, per esempio, dei Chirotteri, per i quali il Centro regionale Chirotteri della Regione Piemonte aveva avviato un'azione di individuazione e caratterizzazione dei siti di svernamento, di riproduzione e di "swarming" e approntato una bozza di Piano d'azione per i Chirotteri del Piemonte nel quale sono indicati i siti di presenza delle specie (Patriarca et al., 2012).

Per quanto riguarda gli Anfibi e i Rettili, oltre all'Atlante Regionale (Andreone \& Sindaco, 1998), era disponibile una valutazione dello stato di conservazione di Pelobates fuscus insubricus in Piemonte con dettagli sul Novarese a cura di Fortina e Marocco (1994). Inoltre, la SHI - Societas Herpetologica Italica aveva individuato, fra le AREN - Aree di rilevanza erpetologica nazionale, il sito ITA027PIE001 Boschi e risaie della Picchetta (http://www-3.unipv.it/webshi/conserv/ areeril.htm).

Per alcuni taxa di invertebrati, per i quali le informazioni erano distribuite in numerose pubblicazioni specialistiche, erano stati recentemente pubblicati atlanti sulla distribuzione a livello regionale (Boano et al., 2007), e provinciale (Riservato, 2009) e approfondimenti su alcune famiglie relativamente a singoli biotopi (Pescarolo, 1993, 1996).

La componente idrobiologica è stata oggetto di numerose ricerche, condotte soprattutto da componenti del CNR-ISE Istituto per lo Studio degli Ecosistemi di Verbania, già Istituto Italiano di Idrobiologia "Dott. Marco De Marchi”.

Infine, per alcuni territori protetti l'investigazione naturalistica era stata più dettagliata. Per esempio, per il Parco del Ticino erano disponibili inventari e aggiornamenti di un certo dettaglio (Furlanetto, 2002, 2002b; Bogliani et al., 2003; Casale et al., 2014).

\section{Il progetto "Novara in rete". La combinazione di due approcci}

L'individuazione delle Aree prioritarie per la conservazione della biodiversità nella provincia di Novara è stata realizzata nell'ambito dell'azione A.3 "Individuazione delle Aree sorgenti di biodiversità" del progetto "Novara in Rete - Studio di fattibilità per la definizione delle Rete Ecologica in Provincia di Novara", coordinato da LIPU BirdLife Italia, in partenariato con Università degli Studi di Pavia, Provincia di Novara, Regione Piemonte e ARPA Piemonte, e cofinanziato da Fondazione CARIPLO. Individuare le Aree prioritarie per la conservazione della biodiversità della provincia di Novara è stato il primo passo per poi disegnare, in una fase successiva, la Rete Ecologica Provinciale su basi naturalistiche. A questa operazione hanno partecipato 26 naturalisti esperti, ossia dotati di competenze personali pregresse e di adeguata conoscenza del territorio della provincia di Novara. Agli esperti è stato chiesto di individuare $\mathrm{i}$ temi focali più rappresentativi per la loro disciplina e di assegnare una valutazione comparativa del valore naturalistico di aree diverse del territorio provinciale. La metodologia adottata, nota come expert-based e basata sull'ottenimento delle informazioni dirette da parte dei maggiori esperti presenti sul territorio, è stata sviluppata per identificare e cartografare le aree più importanti per la conservazione della biodiversità e ha già trovato applicazione nelle Alpi (Arduino et al., 2006), in Lombardia (Bogliani et al., 2007), in Veneto, nella provincia del Verbano-Cusio-Ossola (Bionda et al., 2011), in provincia di Asti (Caprio \& Vazzola, 2011), nei Carpazi, nelle Alpi Dinariche e in altre aree del mondo (Dinerstein et al., 2000).

A una prima ricognizione è apparso che il grado di conoscenza del territorio provinciale da parte degli esperti non era uniforme e che alcune aree erano meno esplorate e conosciute. Per questo motivo, alla metodologia expertbased è stata affiancata una metodologia modellistica, sviluppata a più riprese nell'ultimo decennio da ARPA Piemonte a supporto della valutazione ambientale di piani e progetti (Vietti et al., 2003; Ferrarato et al., 2004; Vietti et al., 2004; Maffiotti et al., 2007; Alibrando et al., 2007; Airaudo et al., 2008); oggetto di una recente e profonda revisione, la metodologia modellistica è stata individuata come strumento utile a definire la rete ecologica regionale dal gruppo di lavoro interdirezionale della Regione Piemonte, istituito con D.G.R. n. 27-7183 del 3 marzo 2014. Applicare anche la metodologia modellistica garantisce la coerenza tra la rete ecologica individuata grazie al progetto "Novara in rete"e la costruenda Rete Ecologica Regionale. Inoltre, consente un ulteriore affinamento e validazione della metodologia expert-based dal momento che il grado di conoscenza del territorio provinciale da parte degli esperti non era omogeneo. In seguito, tramite la metodologia modellistica si sono altresì identificati gli elementi della rete ecologica a partire da dati digitalizzati di uso del suolo e di impatto antropico; di quest'ultimo processo si darà conto in altro lavoro. 


\section{METODI}

\section{Il metodo expert-based}

Nell'ambito dello studio per la provincia di Novara l'obiettivo del metodo expert-based era l'individuazione delle Aree prioritarie per la conservazione della biodiversità. Per l'individuazione e la perimetrazione di tali aree, la raccolta delle informazioni si è basata sul sapere pregresso degli esperti e non ha comportato una nuova raccolta di dati. Il metodo è anche chiamato nella letteratura internazionale expert-based (Dinerstein et al., 2000). Esso presuppone che la conoscenza che già esiste sia sufficiente ad eseguire una analisi generica ma veritiera, e quindi a trarre conclusioni significative. Componenti irrinunciabili del metodo sono perciò gli esperti, il cui sapere si sostituisce in buona parte e/o si aggiunge a rigorose raccolte di dati, impegnative formulazioni di modelli, o approfondite consultazioni di banche dati.

Nelle condizioni riscontrate nel progetto "Novara in Rete", il metodo expert-based offre alcuni vantaggi rispetto a più tradizionali approcci di ricerca:

A) Fornisce informazioni di prima mano, generalmente aggiornate ed elaborate. Gli esperti infatti conoscono il territorio in modo diretto, lo visitano regolarmente e quindi ne notano ogni aspetto e tendenza. Sono in grado di suggerire priorità solo in apparenza basandosi sull'intuito; in realtà fanno riferimento a modelli mentali che sono il frutto di anni di esperienza. Le informazioni ottenute dagli esperti sono pertanto estremamente preziose. La presenza o meno di queste informazioni in letteratura è irrilevante, in quanto solo una minima porzione del sapere di ciascun esperto viene effettivamente travasato nelle pubblicazioni.

B) Porta a risultati in tempi brevi. Dato il ruolo centrale degli esperti e del loro sapere, il metodo permette di condurre analisi e trarre conclusioni in breve tempo, senza ricorrere a estese ricerche.

C) Consente di contenere i costi. Non essendo necessario ricorrere a nuove raccolte di dati, alla creazione di modelli o all'acquisto di banche dati esistenti e, riducendo i tempi, anche i costi sono molto contenuti.

D) Garantisce un controllo scientifico e conferisce legittimità ai risultati. Gli esperti, pur seguendo un metodo che valorizza il quadro conoscitivo soggettivo, non dimenticano il rigore scientifico a cui sono abituati e se ne servono continuamente: lasciano che sia la loro scienza a guidare le loro decisioni. La partecipazione della comunità scientifica conferisce legittimità al processo e validità ai risultati e nello stesso tempo la consultazione degli esperti ne fa dei sostenitori delle conclusioni: gli esperti stessi saranno gli avvocati dei risultati.

E) Conduce a risultati avanzati. Vista la collaborazione fra esperti e il consenso sulle scelte che si realizzano nella fase di consultazione di gruppo, i risultati sono già un'elaborazione più avanzata del lavoro e delle opinioni dei singoli.

F) Offre agli esperti un'occasione unica di scambio e di esperienza. La collaborazione tra esperti richiesta dal metodo expert-based costituisce un'occasione quasi unica di messa in rete, scambio di informazioni, discussione, interdisciplinarietà e acquisizione di esperienza. Nessuno degli esperti, lavorando indipendentemente, potrebbe giungere a una visione d'insieme comparabile e agli stessi risultati avanzati; al contrario, ognuno di essi trae beneficio dal lavoro di gruppo e dalla messa in rete del sapere, in una sinergia altrimenti insperata.

A fronte di questi benefici, il metodo expert-based soffre di una debolezza principale: non è oggettivo, sistematico e ripetibile come altri metodi. Proprio perché si basa sul sapere di un gruppo selezionato e forzatamente ridotto di esperti, risente intrinsecamente di un limite legato all'esperienza soggettiva di quegli esperti. Questo svantaggio tuttavia è meno importante di quel che potrebbe sembrare: in tutte le ecoregioni in cui si è applicato il metodo, a processo terminato, anche gli esperti che non erano stati coinvolti hanno riconosciuto la validità dei risultati ottenuti.

\section{Applicazione del metodo al territorio novarese}

La procedura adottata per l'individuazione delle Aree prioritarie per la biodiversità della provincia di Novara ha seguito le seguenti fasi principali:

1) definizione della scala cartografica di lavoro;

2) definizione dei temi di biodiversità da considerare (taxa, habitat) e conseguente selezione dei gruppi tematici;

3) individuazione degli esperti per i gruppi tematici selezionati;

4) organizzazione degli eventi di consultazione dei gruppi tematici degli esperti;

5) definizione dei criteri per la selezione delle specie o degli habitat focali per i vari temi;

6) definizione dei criteri per l'identificazione delle aree importanti per i vari temi;

7)sovrapposizione dei risultati dei vari gruppi tematici e identificazione delle aree risultate più "ricche" di biodiversità.

I gruppi tematici sono stati definiti in seguito a un'analisi preliminare da parte del gruppo di lavoro della letteratura scientifica, della letteratura "grigia" e delle conoscenze personali, che ha indicato la presenza di esperti qualificati, informati, aggiornati e disposti a partecipare al processo a titolo volontario. I gruppi individuati sono stati i seguenti:

1) Flora e Vegetazione

2) Invertebrati (categoria spuria che includeva prevalentemente ma non esclusivamente Artropodi)

3) Cenosi acquatiche e pesci

4) Anfibi e Rettili

5) Uccelli

6) Mammiferi.

Le consultazioni degli esperti sono avvenute in prima seduta il 22 marzo 2014 presso i locali della Provincia di Novara e sono proseguite con consultazioni telematiche nelle settimane successive. Gli esperti sono stati raggruppati nei rispettivi gruppi tematici alloggiati in sedi diverse e coordinati ciascuno da un componente del gruppo di lavoro del progetto "Novara in Rete" aiutato da un cartografo GIS. A ogni gruppo è stato chiesto di procedere alle seguenti operazioni:

a) identificare specie, habitat e processi focali considerati più rilevanti per il territorio oggetto di studio; 
b) selezionare le aree più importanti per ogni tema; l'area veniva identificata come tale solo se la scelta veniva condivisa da tutti i membri del gruppo tematico, per evitare la selezione di aree aventi solo importanza a livello locale;

c) verificare la rappresentatività delle aree importanti sino a quel momento individuate rispetto ai temi focali definiti all'inizio.

Ai sei gruppi tematici erano stati forniti i criteri da utilizzare nel processo di identificazione, da indicare in apposite schede per ciascuna delle aree importanti. In particolare si chiedeva che, affinché un'area potesse essere identificata come importante, venissero soddisfatti uno o più dei seguenti requisiti:

1) presenza di specie, habitat, cenosi, ambiti o processi ecologici focali;

2) ricchezza di specie, di habitat o di processi ecologici a livello di ecoregione o continentale;

3) presenza di endemismi;

4) presenza di specie della Direttiva Uccelli (solo per il gruppo tematico "Uccelli");

5) presenza di specie della Direttiva Habitat;

6) presenza di habitat d'interesse comunitario della Direttiva Habitat (per il gruppo tematico "Flora e vegetazione").

Inoltre, quando gli esperti di ciascun gruppo tematico avevano appena concluso l'identificazione delle aree importanti, il coordinatore chiedeva di indicare quali tra le aree indicate fossero "peculiari" o "imprescindibili", cioè così importanti da meritare di divenire prioritarie anche se nessun altro gruppo tematico le avesse identificate come importanti. Tali aree peculiari dovevano essere in numero ridotto; talvolta una sola.

In seguito, si è proceduto con la sovrapposizione degli strati cartografici relativi alle aree importanti dei diversi gruppi tematici, e si sono individuate le aree prioritarie laddove ci fossero ricorrenze di poligoni. Per poligono si intende una qualsiasi figura geometrica delimitata da una linea spezzata chiusa i cui vertici siano stati localizzati nel piano attraverso un programma di GIS; i poligoni sono associati a una tabella con gli attributi del territorio incluso.

$\mathrm{Si}$ è tenuto conto della necessità di non indicare tutte le aree interessate da un solo poligono; in questo caso, una parte rilevante del territorio provinciale sarebbe stata classificata come prioritaria, non consentendo di designare i poligoni più ricchi. D'altra parte, non si è ritenuto di spingersi a considerare come aree prioritarie solo i poligoni risultanti dalla sovrapposizione di tutti i 6 gruppi tematici o anche solo da 4 o 5, in quanto, in questo modo, sarebbero state escluse porzioni importanti del territorio comunque ricche di valore per un numero significativo di gruppi indicatori.

\section{Il metodo modellistico}

Nell'ambito dello studio per la provincia di Novara l'obiettivo del metodo modellistico era la verifica della corrispondenza fra quanto sarebbe emerso con il metodo naturalistico expert based e l'analisi attraverso l'uso di database dell'uso del suolo attraverso opportuni approcci di calcolo. Il progetto "Novara in rete", infatti, potrebbe essere propedeutico alla realizzazione di un progetto di rete ecologica complessiva della regione Piemonte.

I modelli ecologici elaborati attraverso il calcolo automatico permettono di valutare con un criterio oggettivo la presenza di Aree di Valore Ecologico (AVE d'ora in poi) e altre con funzione di corridoio ecologico, creando così un sistema dinamico volto a tutelare le aree a maggior biodiversità e le aree residuali potenzialmente utilizzabili a seguito di interventi di potenziamento e/o ripristino delle connessioni ecologiche.

I principali passi metodologici seguiti per la realizzazione del prodotto finale delle AVE sono stati:

- predisposizione della base dati cartografica di riferimento;

- elaborazione della carta degli habitat;

- realizzazione di un database per alcune specie di mammiferi, uccelli ed invertebrati inclusi nella Direttiva 92/43/CEE "Habitat" e nella Direttiva 2009/147/CE "Uccelli", presenti in provincia di Novara e valutazione delle affinità specie-habitat per ciascuna di esse;

- elaborazione degli indicatori faunistici per mammiferi, uccelli ed alcuni invertebrati inclusi negli allegati 2 e 4 della Direttiva Habitat,

- elaborazione degli indicatori vegetazionali;

- individuazione delle Aree di Valore Ecologico.

L'intero processo di individuazione delle AVE e di creazione della rete ecologica è stato studiato per poter essere riproducibile con la cartografia esistente e con software libero; i software utilizzati sono stati: Grass, Qgis, Postgres/Postgis.

La procedura adottata per l'individuazione della rete ecologica della provincia di Novara è stata strutturata seguendo le fasi di seguito descritte.

\section{Predisposizione della base dati di riferimento}

La base dati di partenza è costituita dal LandCover Piemonte (LCP) 2010, integrata, per alcuni aspetti, da informazioni desumibili da banche dati più recenti o di maggior dettaglio:

- prati stabili (dall'anagrafe agricola regionale)

- habitat forestali (dai Piani Forestali territoriali)

- idrografia (dal grafo idrico regionale)

- tipologie stradali (dal grafo stradale regionale)

\section{Elaborazione della carta degli habitat}

Per la realizzazione della carta degli habitat si è adottato il sistema di classificazione EUNIS, sviluppato dall'Agenzia Europea per l'Ambiente (http://eunis.eea.europa.eu/). In funzione del dettaglio che le basi dati disponibili consentono di raggiungere, sono state selezionate in via preliminare 73 tipologie ambientali, di cui 54 effettivamente presenti sul territorio provinciale. Le tipologie selezionate costituiscono la legenda di riferimento e corrispondono, per la maggior parte delle tipologie di ambienti naturali o semi-naturali, almeno al terzo livello della classificazione adottata: a queste sono state ricondotte tutte le voci della legenda LCP. Sulla base di tale legenda è stata creata la carta degli habitat della provincia di Novara. 
Realizzazione di un database per tutte le specie di mammiferi, uccelli e invertebrati in Direttiva Habitat presenti in provincia di Novara e valutazione delle affinità specie-habitat per ciascuna di esse

Il modello è stato esteso ad alcuni taxa sicuramente presenti nell'area che si intendeva indagare, suddivisi nelle seguenti categorie sistematiche: Mammiferi; Uccelli; alcuni invertebrati inclusi negli Allegati II/IV della Direttiva 92/43/CEE "Habitat" (Lepidotteri e Coleotteri Carabidi).

Per ciascuna specie è stata effettuata un'analisi speciehabitat che metteva in relazione le caratteristiche ambientali con le esigenze ecologiche specifiche attribuendo il grado di affinità dei differenti habitat in termini di potenzialità di risorse disponibili per ciascuna specie. A tal fine, si procede attribuendo un valore in un intervallo compreso tra 0 e 1 sulla base delle relazioni esistenti tra la specie esaminata e le categorie di habitat presenti. Il valore 0 indica ambienti non idonei per la presenza della specie studiata, mentre il valore 1 identifica gli ambienti ad alta idoneità.

È stato quindi messo a punto un database con le informazioni per le specie considerate, il cui utilizzo ha reso possibile lo sviluppo di indicatori che hanno consentito di individuare le aree di valore ecologico per la fauna. Infine, utilizzando il modello di affinità specie-habitat, sono state allestite mappe preliminari per ciascuna specie dove l'unico parametro indicato è l'idoneità dell'habitat.

\section{Elaborazione degli indicatori faunistici per mammiferi, uccelli e invertebrati di interesse conservazionistico}

Collegando il database delle specie alla carta degli habitat sono state elaborate le cartografie relative all'idoneità specie-habitat per i tre gruppi considerati; una volta individuate le aree a diverso grado di idoneità, estraendo unicamente quelle ad alta idoneità per ciascun gruppo sistematico sono state individuate:

- aree importanti per i Mammiferi;

- aree importanti per gli Uccelli;

- aree importanti per alcuni Artropodi inclusi negli allegati della Direttiva "Habitat" (Lepidotteri e Coleotteri Carabidi).

\section{Elaborazione degli indicatori vegetazionali}

L'analisi vegetazionale è stata condotta applicando un set di quattro indicatori finalizzati a valutare, per ciascuno degli ambienti considerati: 1) la distanza dal climax, 2) la naturalità, qui intesa come l'inverso del livello di determinismo antropico, 3) il valore floristico, sulla base di quanto desumibile da letteratura, e 4) l'importanza conservazionistica, ovvero la possibile inclusione di un determinato ambiente nell'allegato I della Direttiva 92/43/CEE "Habitat". I quattro indicatori sono stati poi aggregati in un unico indice sintetico (Is), attribuendo a ciascuno di essi un diverso peso, secondo lo schema seguente:

Is $=0,8 *$ climax $+0,8 *$ naturalità $+0,5 *$ biodiversità + $0,3 *$ conservazionistico.
Si è ritenuto di selezionare tutti gli habitat della legenda di riferimento che abbiano un valore di Is superiore a quello attribuito all'ambiente "Boschi e foreste di $\mathrm{Ca}$ stanea sativa" (cod. EUNIS G1.7D) come concorrenti a individuare le aree di valore ecologico.

\section{Gap analysis}

La gap analysis, traducibile come la comparazione della "performance" attuale con quella potenziale, è stata condotta confrontando le sovrapposizioni delle Aree prioritarie per la biodiversità individuate con questo studio con le aree protette esistenti e con altre categorie di tutela del territorio. Questa procedura consente di valutare in modo oggettivo l'efficacia di questi metodi per la conservazione della biodiversità. Infatti, mentre le Aree prioritarie sono state identificate per la tutela della biodiversità, le altre categorie di gestione del territorio non sempre hanno questo obiettivo, con l'eccezione di parchi e riserve naturali, SIC, ZPS. La gap analysis diventa quindi opportuna per evidenziare eventuali carenze nei sistemi di tutela e di pianificazione esistenti e suggerire correzioni o integrazioni. Le integrazioni non vanno intese solo come creazione di nuove aree protette, ma anche come adozione di appropriate forme di gestione di territori in cui natura e attività umane coesistono in maniera complessa.

\section{RISULTATI}

\section{Individuazione delle Aree prioritarie per la biodiversità con il metodo expert based}

Ciascun gruppo tematico di lavoro ha proceduto autonomamente individuando preliminarmente i propri temi focali e giungendo infine a identificare e definire cartograficamente le aree importanti per il tema di competenza. Di seguito vengono presentati gli esiti di quanto emerso dai singoli gruppi tematici. Ciascuna area è definita da una sigla e dal nome per esteso. I nomi in corsivo indicano le aree classificate come peculiari.

\section{Flora e vegetazione}

Il gruppo tematico Flora e vegetazione ha complessivamente identificato 17 Aree importanti (Fig. 1).

FL01 Agogna Morta

FL02 Campo della Ghina

FL03 Baraggia di Bellinzago

FL04 Baraggia di Piano Rosa

FL05 Monte Fenera

FL06 Valle del Ticino

FL07 Lagoni di Mercurago

FL08 Canneti di Dormelletto

FL09 Lago d'Orta (area peculiare)

FL10 Fontanili a nord di Novara (area peculiare)

FL11 Risaie tra Casalino e Granozzo

FL12 Torrente Vevera (area peculiare)

FL13 Torrente Agogna (area peculiare)

FL14 Alpe della Volpe

FL15 Bosco Preti

FL16 Torbiera Agrate Conturbia (area peculiare)

FL17 Rocca di Arona 


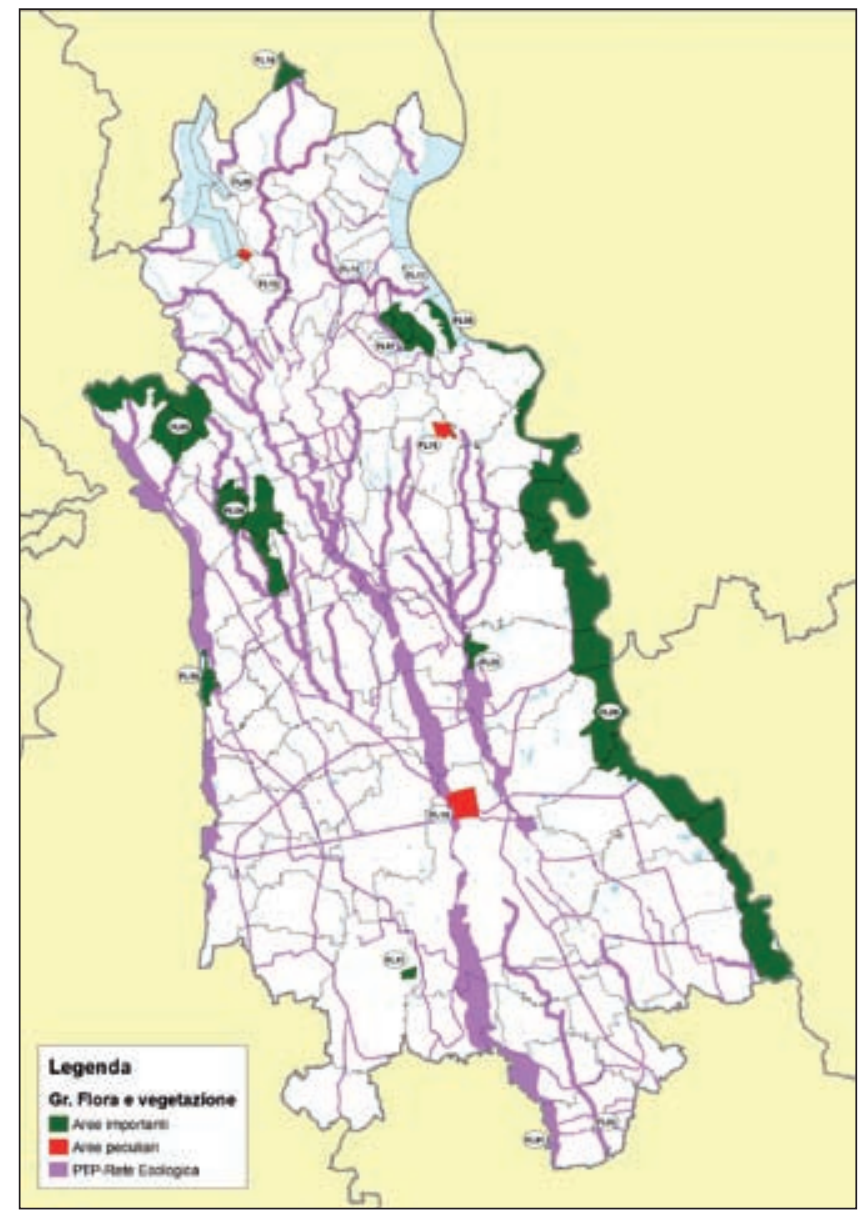

Fig. 1 - Aree importanti individuate dagli esperti del gruppo tematico Flora e vegetazione.

\section{Invertebrati}

Il gruppo tematico Invertebrati ha complessivamente identificato 21 Aree importanti (Fig. 2).

IN01 Palude di Casalbeltrame

IN02 Roggia Busca e Roggia Biraga, Quintino Sella

IN03 Canale Cavour (area peculiare)

IN04 Ticino (area peculiare)

IN05 Burchvif (area peculiare)

IN06 Cascina Valtoppa

IN07 Valle dell'Arbogna (area peculiare)

IN08 Baragge

IN09 Risaie di Sozzago e Tornaco (area peculiare)

IN10 Fiume Sesia (area peculiare)

IN11 Monte Fenera (area peculiare)

IN12 Alto Sizzone e Cremosina

IN13 Alto Agogna

IN14 Lagoni di Mercurago

IN15 Valle del Pescone

IN16 Forre del Vevera

IN17 Alto Vergante

IN18 Baraggia di Bellinzago

IN19 Fontanili alti

IN20 Fontanili bassi

IN21 Torbiera di Agrate Conturbia

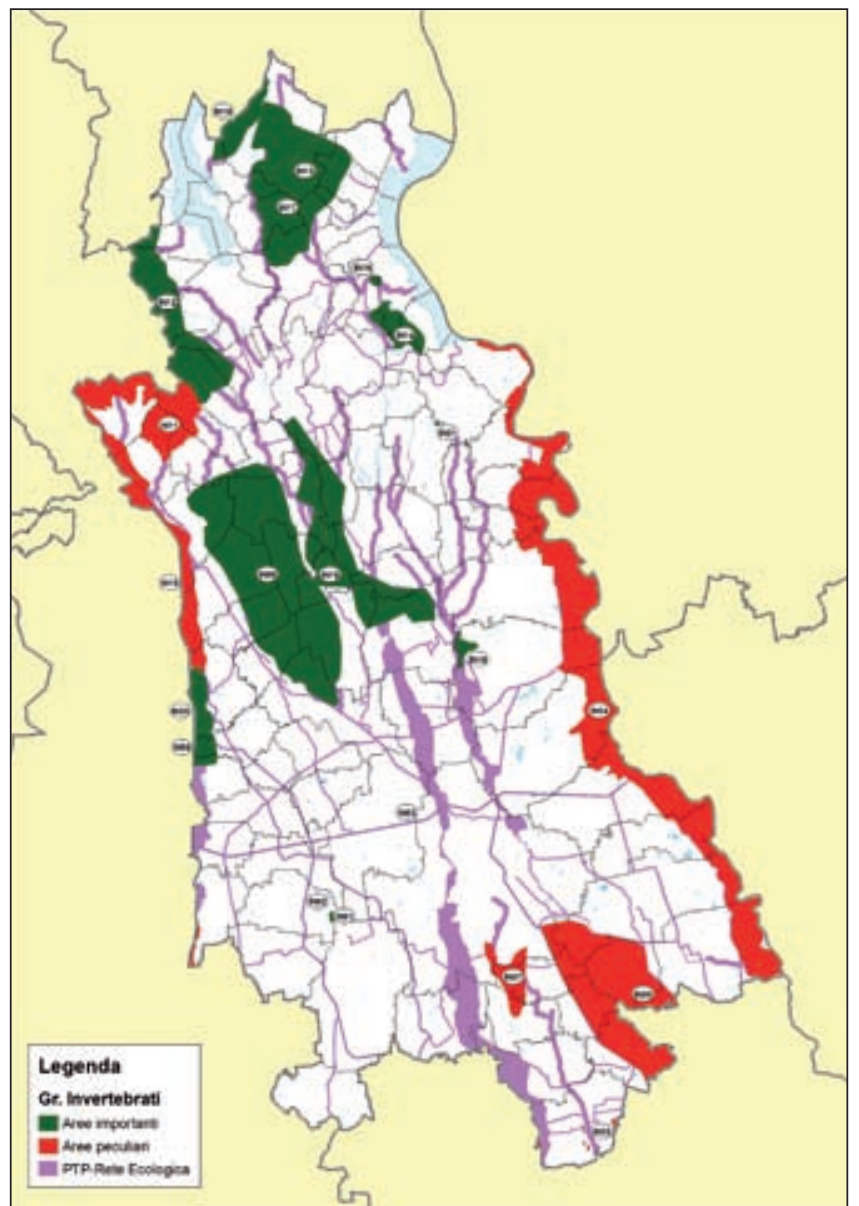

Fig. 2 - Aree importanti individuate dagli esperti del gruppo tematico Invertebrati.

Cenosi acquatiche e pesci

Il gruppo tematico Cenosi acquatiche e pesci ha complessivamente identificato 14 Aree importanti (Fig. 3).

CEN01 Foce torrente Erno

CEN02 Canneti di Dormelletto

CEN03 Lagoni di Mercurago

CEN04 Foce torrente Pescone

CEN05 Foce torrente Qualba

CEN06 Lago d'Orta (area peculiare)

CEN07 Fiume Ticino

CEN08 Lago Maggiore

CEN09 Torrente Terdoppio

CEN10 Roggia Mora (area peculiare)

CEN11 Torrente Agogna

CEN12 Fiume Sesia

CEN13 Canale Cavour

CEN14 Fontanili e risorgive

\section{Anfibi e rettili}

Il gruppo tematico Anfibi e Rettili ha complessivamente identificato 16 Aree importanti (Fig. 4).
ERP01
Zone baraggive
ERP02 Zone baraggive
1
ERP03
Zone baraggive
ERP04 Zone baraggive 


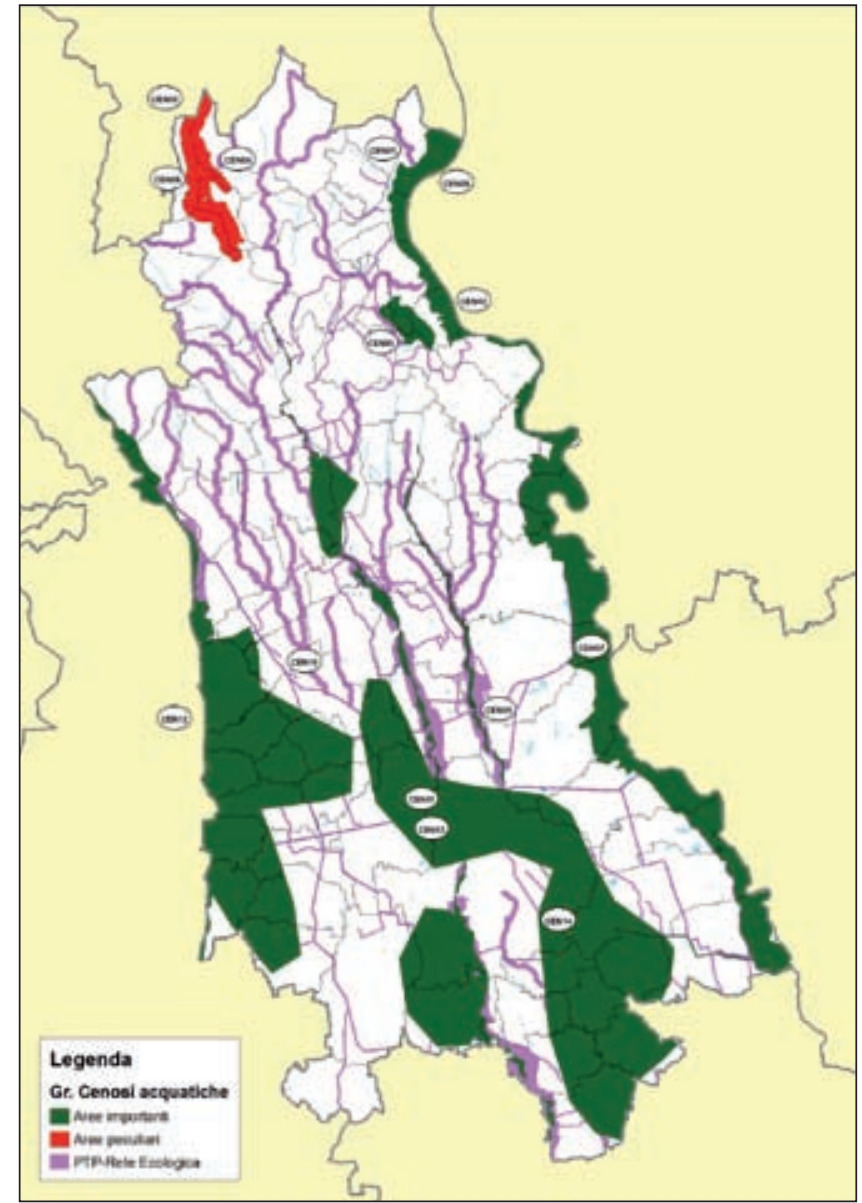

Fig. 3 - Aree importanti individuate dagli esperti del gruppo tematico Cenosi acquatiche e pesci.

ERP05 Fascia ripariale torrente Agogna

ERP06 Zona dei fontanili 1

ERP07 Zona dei fontanili 2

ERP08 Zona dei fontanili 3

ERP09 Zona dei fontanili 4

ERP10 Fascia ripariale fiume Sesia

ERP11 Casalbeltrame

ERP12 Lagoni di Mercurago

ERP13 Zone baraggive 5

ERP14 Fascia ripariale fiume Ticino

ERP15 Mottarone

ERP16 Agogna morta

\section{Uccelli}

Il gruppo tematico Uccelli ha complessivamente identificato 37 Aree importanti (Fig. 5).

UC01 Garzaie del Novarese e Risaie di Barengo

UC02 Garzaia di Cascina Rosa

UC03 Garzaia di Casalbeltrame

UC04 Garzaia di Casalino

UC05 Risaie e Garzaia di Granozzo

UC06 Garzaie di Nibbiola e Vespolate

UC07 Risaie di Sozzago

UC08 Boschi di "Burchvif"

UC09 Boschi 2080 di Novara

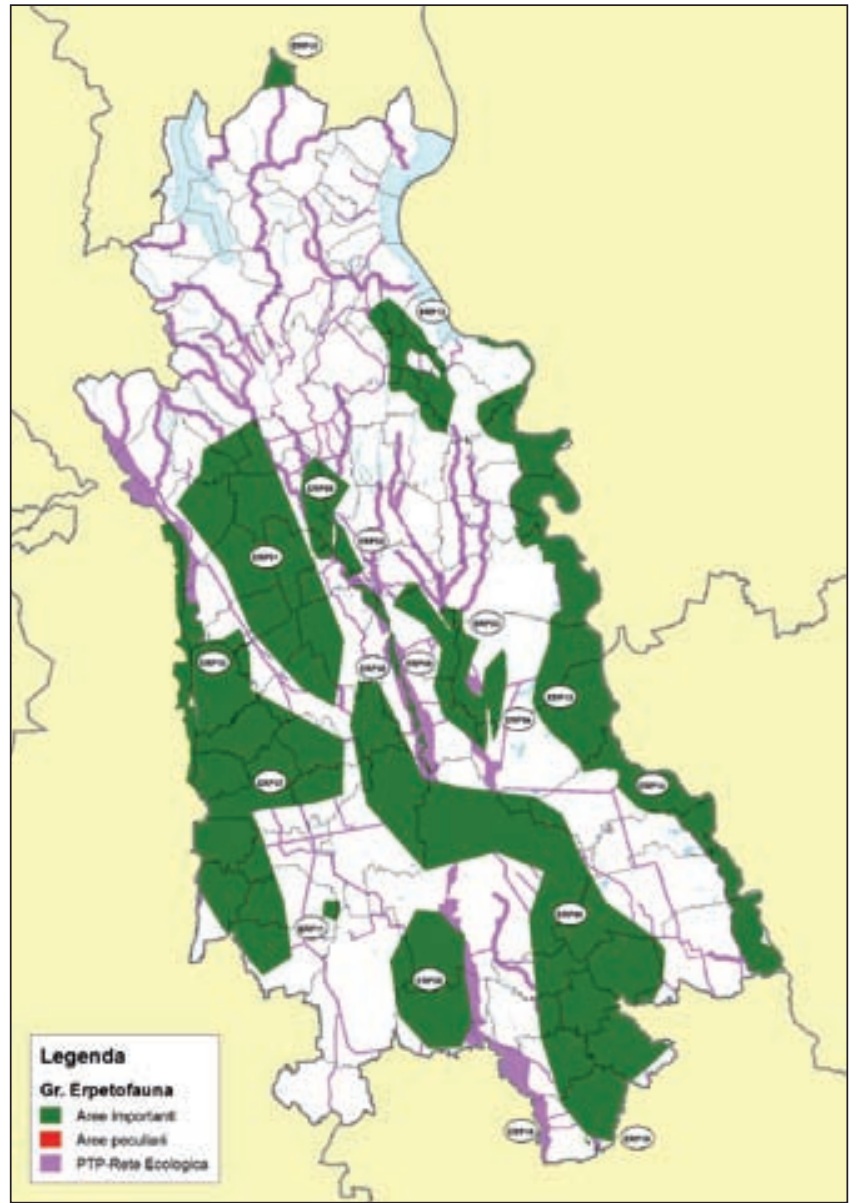

Fig. 4 - Aree importanti individuate dagli esperti del gruppo tematico Anfibi e Rettili.

UC10 Collina di Barengo

UC11 Bosco di Agognate

UC12 Palude di Casalbeltrame (area peculiare)

UC15 Porzione di Torrente Agogna (area peculiare)

UC16 Cava Teodora (area peculiare)

UC17 Linduno e Badia di Dulzago

UC18 Asta del Fiume Sesia

UC19 Fiume Agogna

UC20 Torrente Terdoppio

UC21 Fiume Ticino

UC22 Nido di Cicogna di Romentino

UC23 Nido di Cicogna di Cerano

UC24 Nido di Cicogna di Terdobbiate

UC25 Canneti del Lago d'Orta

UC26 Canneti del Lago d'Orta sud

UC27 Lago Maggiore

UC28 Canneti di Dormelletto

UC29 Lagoni di Mercurago

UC30 Bosco Solivo

UC31 Piano Rosa

UC32 Bosco della Panigà

UC34 Valle dell'Arbogna

UC35 Baragge di Cameri

UC36 Bosco della Bindillina

UC37 Monte del Falò 


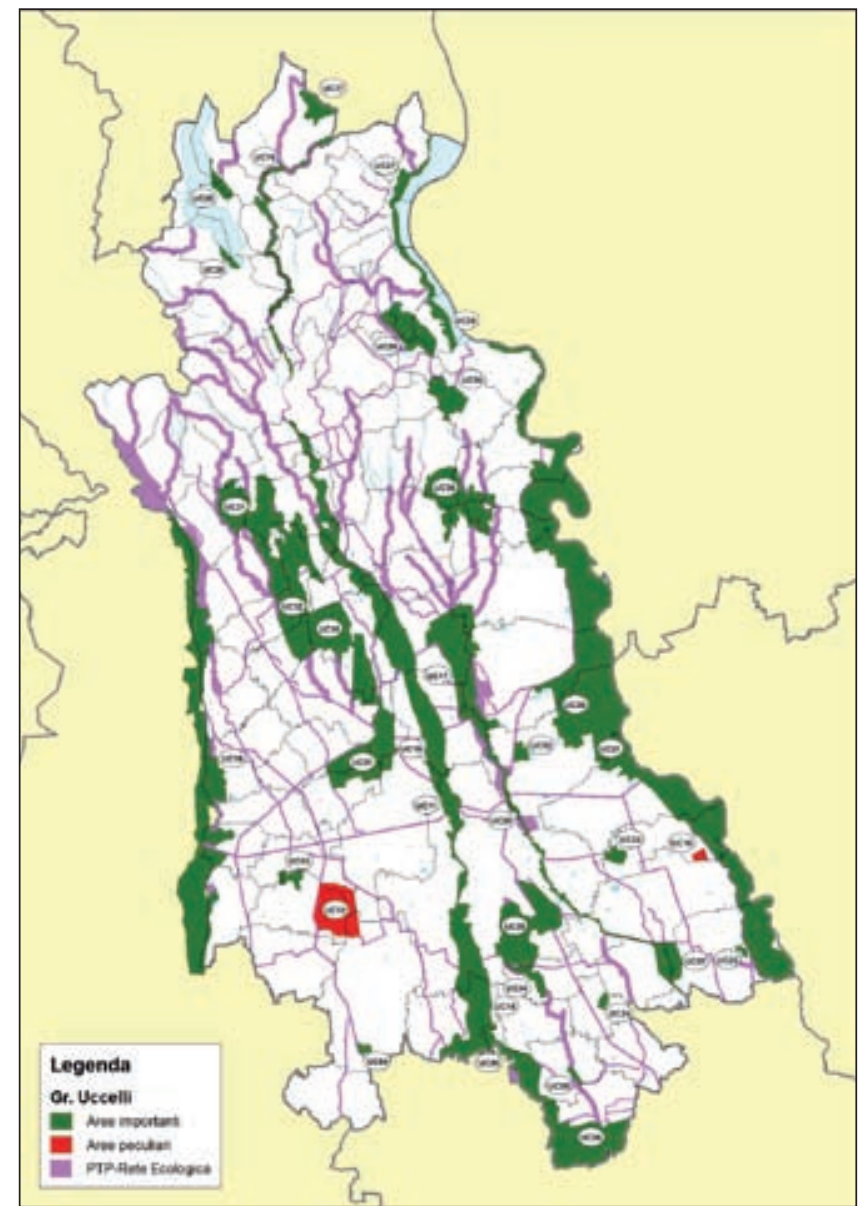

Fig. 5 - Aree importanti individuate dagli esperti del gruppo tematico Uccelli.

\section{Mammiferi}

Il gruppo tematico Mammiferi ha complessivamente identificato 15 Aree importanti (Fig. 6).

M01 Praterie montane di Armeno, Sovazza e Coiromonte

M03 Canneti del Lago d'Orta

M04 Canneti di Dormelletto

M06 Lagoni di Mercurago

M05 Bosco Solivo

M07 Colline moreniche fra Gattico e Canova

M09 Piano Rosa (area peculiare)

M13 Dossi di Borgolavezzaro

M14 Area del Torrente Arbogna

M12 Golene dell'Agogna a valle di Borgomanero (area peculiare)

M11 Golene della Sesia

M02 Alta Valle Agogna

M15 Brughiera di Cameri

M10 Valle del Ticino

M08 Monte Fenera

\section{Individuazione della aree prioritarie con il metodo expert based}

La sovrapposizione degli strati cartografici relativi alle aree importanti definite da ciascun gruppo tematico

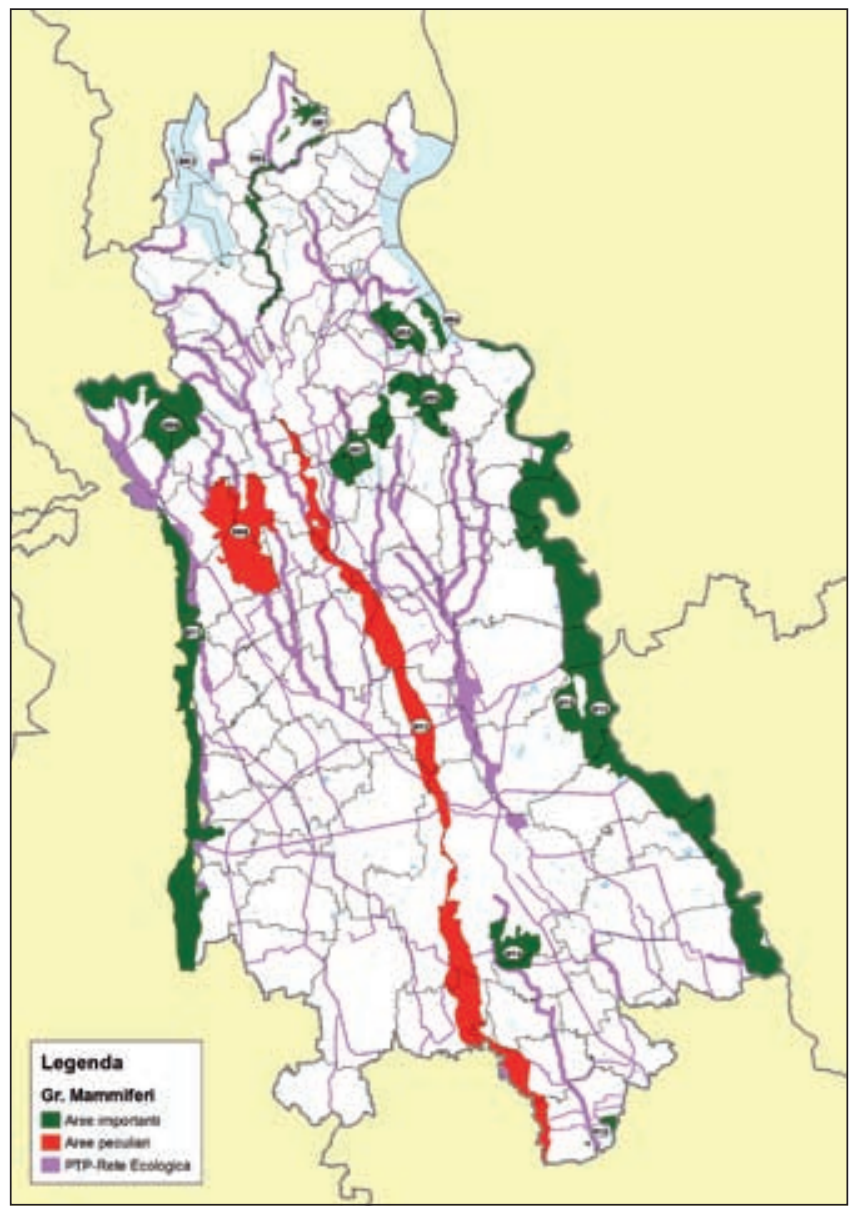

Fig. 6 - Aree importanti individuate dagli esperti del gruppo tematico Mammiferi.

ha portato a una prima definizione delle Aree prioritarie. Un'analisi visuale delle aree risultanti dall'utilizzo di numeri diversi di strati ha mostrato che la sovrapposizione di soli due strati avrebbe incluso una superficie di territorio eccessivamente vasta, comprendente ampie aree di coltivazioni intensive nella bassa pianura di basso valore naturale. D'altro canto, con questo criterio sarebbero incluse estensioni significative della parte collinare-montana che, al contrario, resterebbero escluse con criteri più selettivi (Fig. 7). La sovrapposizione di tre strati sembra essere ottimale per l'area di pianura ma eccessivamente penalizzante per le aree collinaremontane (Fig. 8). La scelta di criteri ancora più selettivi, con 4 e 5 strati, avrebbe portato all'esclusione di zone importanti dell'alta pianura (Figg. 9 e 10). La scelta è pertanto caduta sui valori di 3 strati per l'area di pianura e di 2 strati per l'area di provincia inclusa nel territorio della "Convenzione delle Alpi". Questa differenza deriva dal diverso grado di copertura delle ricerche per i due territori e dalla necessità di integrare le delimitazioni delle Aree prioritarie dell'area alpina con quelle già designate per la provincia del VerbanoCusio-Ossola (Bionda et al., 2011) e più in generale con le Alpi, come risultanti dal lavoro di Arduino et al. (2006), per le quali era stato utilizzato il valore di 2 strati sovrapposti. 


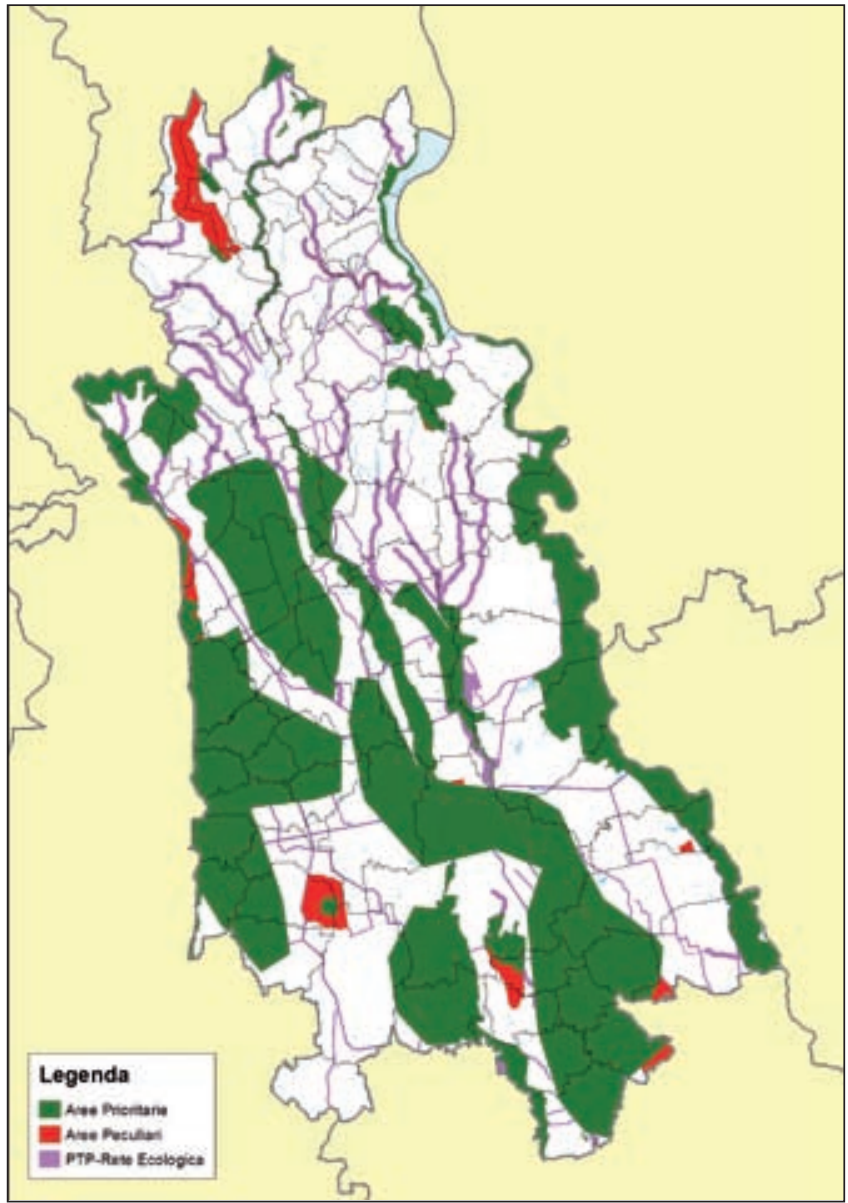

Fig. 7 - Territori individuati nel caso in cui si sovrappongono i poligoni di Aree importanti delimitate da 2 gruppi tematici. In verde sono delimitati i poligoni risultanti dalla semplice sovrapposizione delle Aree importanti; in rosso sono evidenziati i poligoni delle Aree peculiari. La base della Rete Ecologica Provinciale vigente serve per i riferimenti cartografici.

Complessivamente, sono state individuate 23 Aree prioritarie per la biodiversità (Fig. 11 e Tab. 1). Le caratteristiche di ciascuna Area prioritaria sono descritte in Appendice 1.

\section{Individuazione delle Aree di Valore Ecologico con il metodo modellistico}

Per individuare e delimitare le Aree di Valore Ecologico, AVE di seguito, si è proceduto selezionando gli ambienti che soddisfano uno dei due seguenti criteri: a) essere importanti per la vegetazione e per almeno uno dei tre gruppi faunistici, oppure, b) essere importanti per tutti e tre i gruppi faunistici.

Le aree occupate dai 36 habitat (dei 73 inclusi nella legenda di riferimento) così individuati sono state poi integrate con alcune tipologie di ambienti (torbiere, stagni e lanche) incluse nella Banca Dati delle Zone Umide del Piemonte (http://www.regione.piemonte.it/ ambiente/tutela_amb/zu.htm). In questo modo è stato possibile individuare le AVE per la provincia di Novara (Fig. 12).

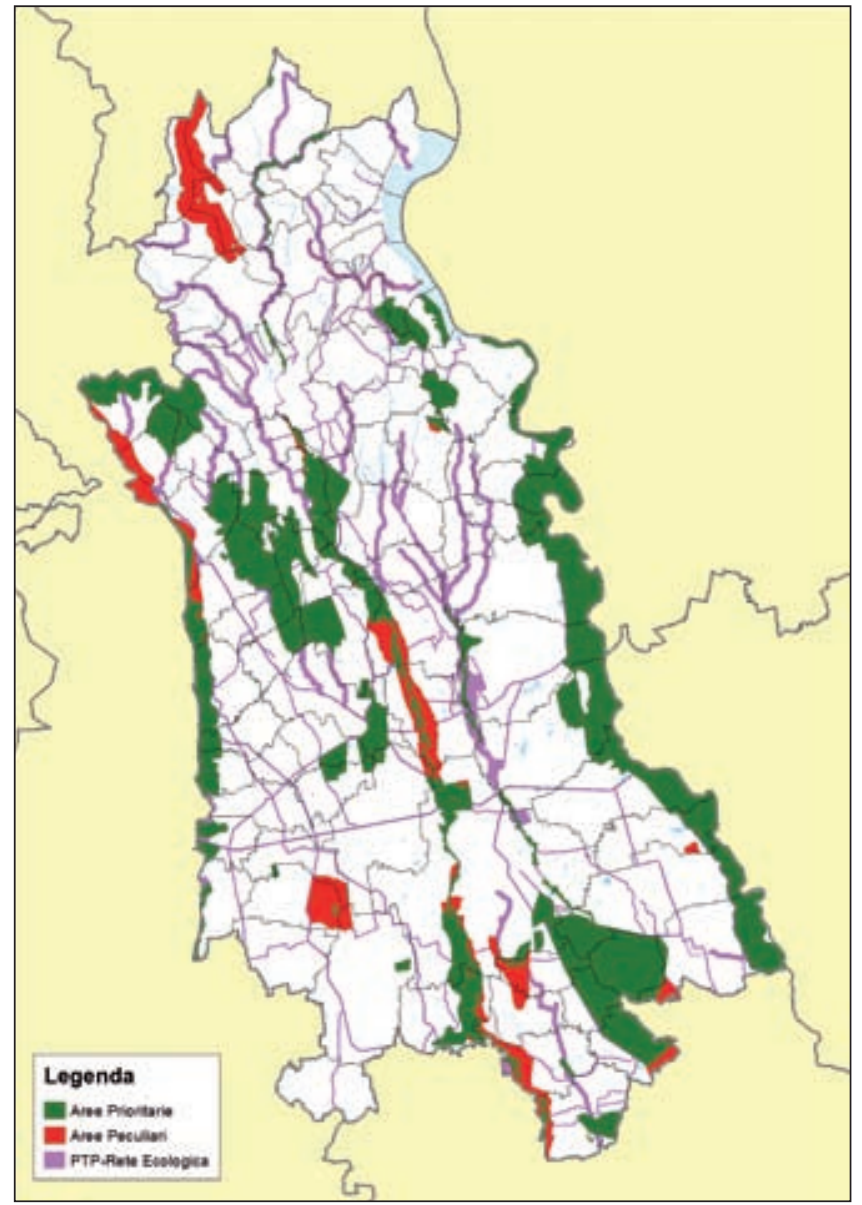

Fig. 8 - Territori individuati nel caso in cui si sovrappongono i poligoni di Aree importanti delimitate da 3 gruppi tematici. In verde sono delimitati i poligoni risultanti dalla semplice sovrapposizione delle Aree importanti; in rosso sono evidenziati i poligoni delle Aree peculiari. La base della Rete Ecologica Provinciale vigente serve per i riferimenti cartografici.

La distribuzione delle AVE mostra una forte dicotomia tra il settore settentrionale e quello meridionale del territorio provinciale; mentre il primo è caratterizzato da numerose AVE e da una matrice territoriale altamente permeabile per le specie considerate, il secondo, contraddistinto soprattutto da vaste superfici a risaia e solo secondariamente da altre colture intensive, è caratterizzato da una presenza sporadica di AVE e da un livello di permeabilità generalmente nullo o molto basso per le specie considerate nei modelli, con elevata frammentazione delle tessere residue, che risultano quindi scarsamente connesse tra loro (Fig. 12). Notevole la presenza di due aree che si configurano come corridoi principali con andamento Nord-Sud, costituite dalle fasce fluviali dei fiumi Ticino e Sesia, che presentano un buon grado di funzionalità, mentre un terzo corridoio, lungo il corso del Torrente Agogna, si presenta piuttosto discontinuo e, soprattutto a Sud del capoluogo, molto frammentato. Tali indicazioni possono rivestire una certa utilità nella pianificazione di eventuali interventi di "deframmentazione" del territorio. 


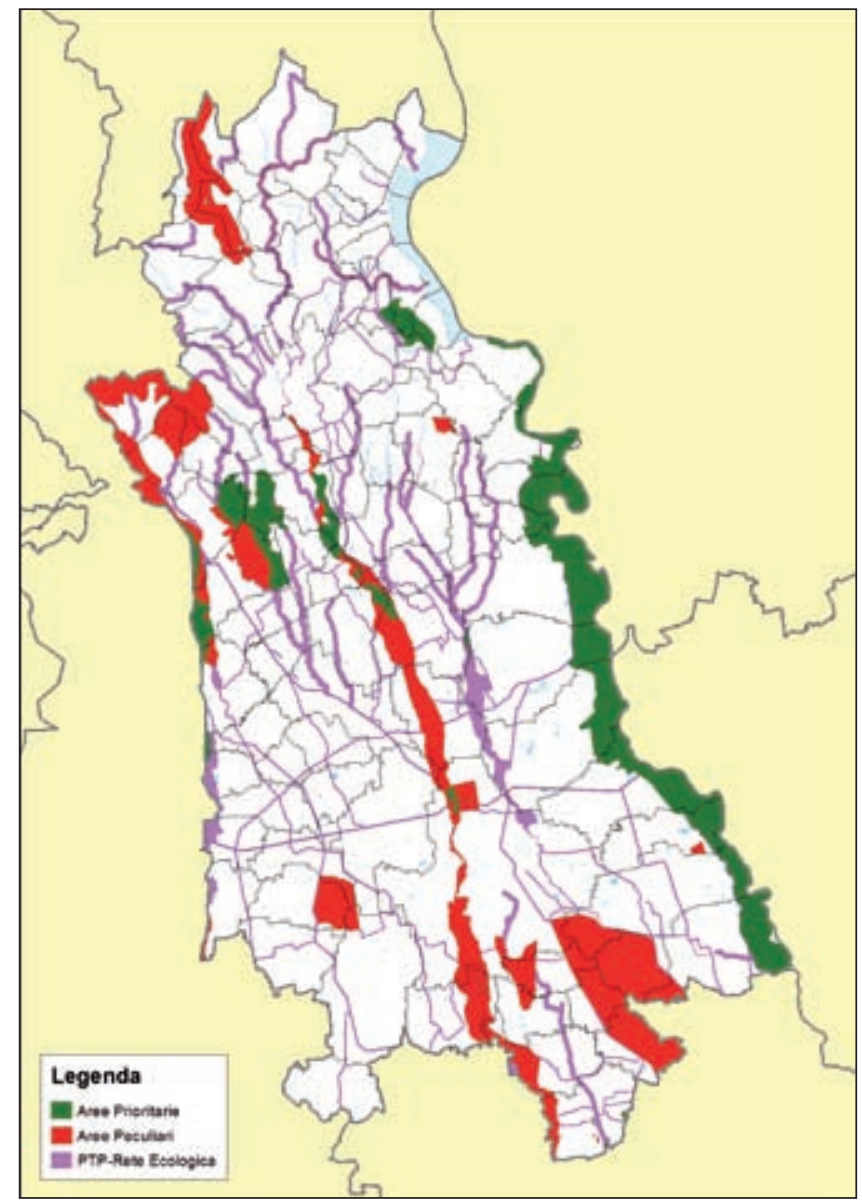

Fig. 9 - Territori individuati nel caso in cui si sovrappongono i poligoni di Aree importanti delimitate da 4 gruppi tematici. In verde sono delimitati i poligoni risultanti dalla semplice sovrapposizione delle Aree importanti; in rosso sono evidenziati i poligoni delle Aree peculiari. La base della Rete Ecologica Provinciale vigente serve per i riferimenti cartografici.

Come già emerso dalla sperimentazione del modello in altre realtà del Piemonte, si evidenzia come in alcuni casi la qualità ed il dettaglio delle basi dati utilizzate abbiano determinato una certa sottostima nell'individuazione delle AVE, soprattutto per quanto riguarda gli ambienti naturali o semi-naturali non forestali (p.e. brughiere o zone umide).

\section{Risultati della gap analysis}

La gap analysis delle Aree prioritarie confrontate con le aree protette e con altre categorie di tutela del territorio ha permesso di valutare l'efficacia di questi sistemi per la conservazione della biodiversità. Infatti, mentre le Aree prioritarie sono state identificate per la loro biodiversità, le altre categorie di tutela (ad eccezione di parchi e riserve naturali, SIC, ZPS) non hanno questo obiettivo: la gap analysis diventa quindi strumento essenziale per evidenziare eventuali carenze nei sistemi di tutela esistenti e suggerire correzioni o integrazioni. Le integrazioni non vanno sempre nella direzione della creazione di nuove aree protette, ma possono suggerire l'adozione di appropriate forme di gestione di territori in

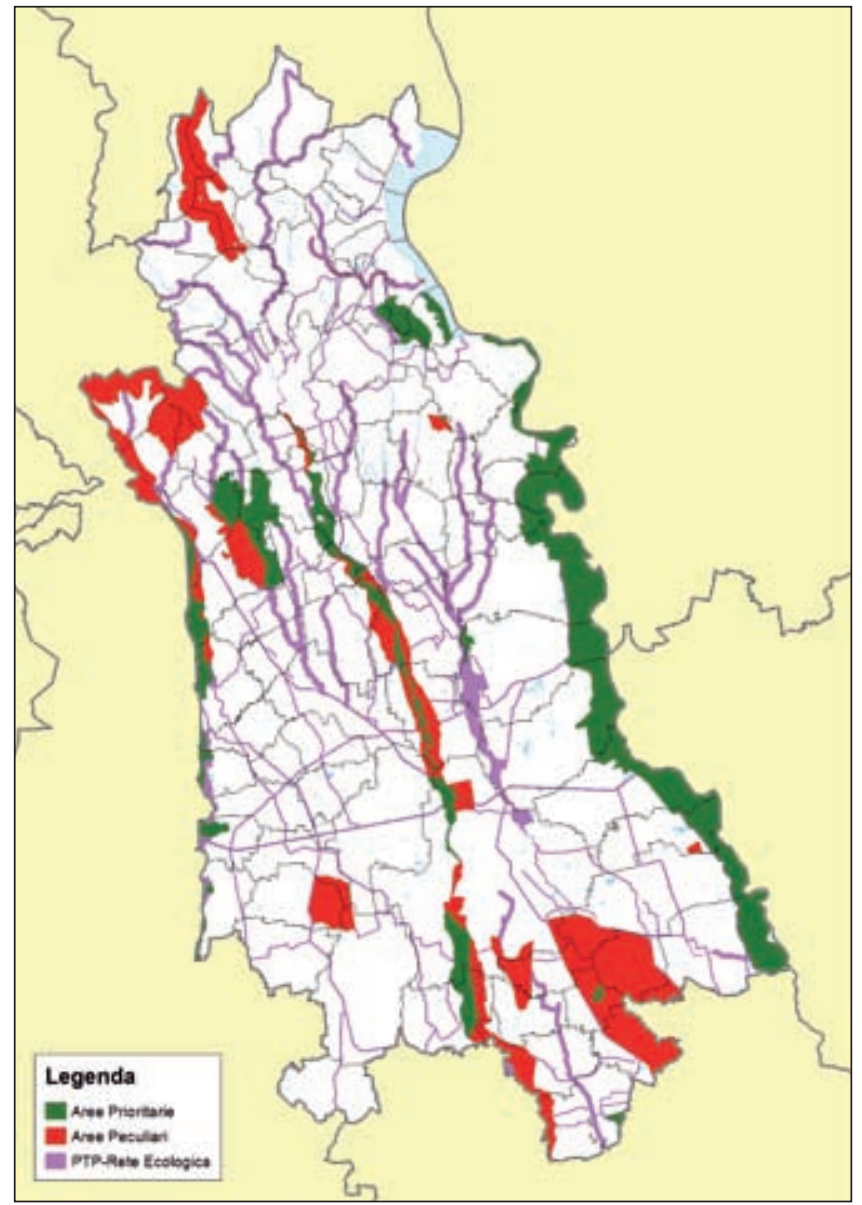

Fig. 10 - Territori individuati nel caso in cui si sovrappongono i poligoni di Aree importanti delimitate da 5 gruppi tematici. In verde sono delimitati i poligoni risultanti dalla semplice sovrapposizione delle Aree importanti; in rosso sono evidenziati i poligoni delle Aree peculiari. La base della Rete Ecologica Provinciale vigente serve per i riferimenti cartografici.

cui natura e attività umane coesistono in maniera complessa. Le Aree protette individuate, i SIC e le ZPS sono elencate in Tab. 2. Le sovrapposizioni fra le Aree Prioritarie, le Aree Protette, i SIC e le ZPS sono evidenziate nelle Figg. 13, 14 e 15.

\section{DISCUSSIONE}

Le 23 Aree Prioritarie per la Biodiversità e le Aree di Valore Ecologico individuate in provincia di Novara disegnano un quadro oggettivo della biodiversità di questo territorio, in passato relativamente poco considerato o valutato solo sulla base di indicatori parziali. La pluralità degli indicatori considerati consente ora di conoscere e di adottare misure di gestione appropriate non falsate da approcci eccessivamente specialistici.

I due metodi di lavoro utilizzati, la valutazione $e x-$ pert based e la delimitazione con l'approccio modellistico delle AVE, hanno portato a identificare aree solo in parte sovrapposte. Le sovrapposizioni si verificano nelle aree di interesse naturalistico individuate dagli esperti che sono anche caratterizzate da valori elevati dei poligoni ricavati con il metodo modellistico; questo avviene 


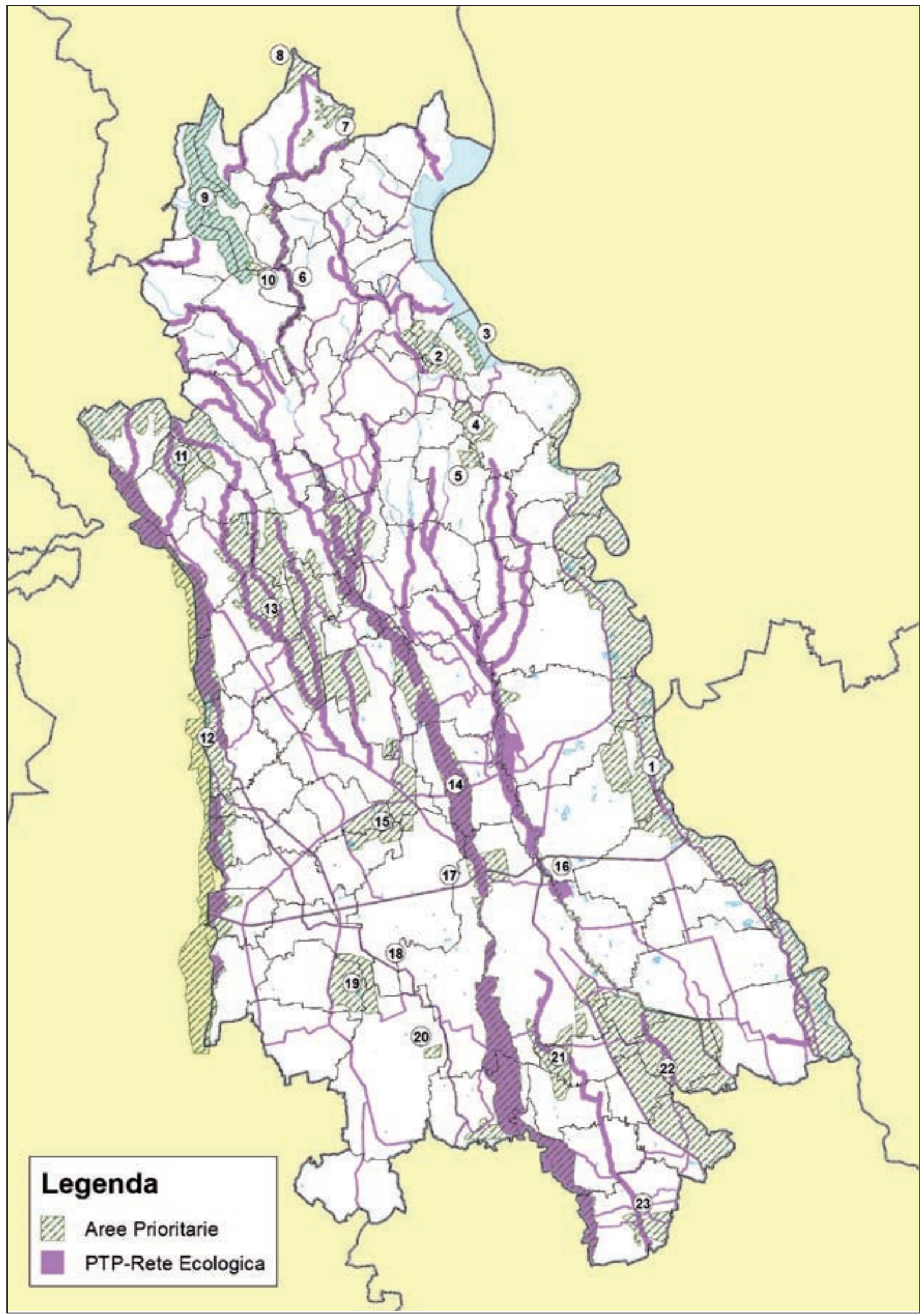

Fig. 11 - Delimitazioni delle 23 Aree prioritarie per la biodiversità della provincia di Novara definite sulla base della sovrapposizione di almeno 3 strati di poligoni delle Aree importanti, oltre ai poligoni delle Aree peculiari. La base della Rete Ecologica Provinciale vigente serve per i riferimenti cartografici. La numerazione delle Aree prioritarie per la biodiversità è la stessa della Tab. 1 . 
Tab. 1 - Aree prioritarie per la biodiversità della provincia di Novara.

\begin{tabular}{|c|c|c|}
\hline ID & TEMATISMI INTERESSATI & DENOMINAZIONE \\
\hline 1 & UC21-35/M10-15/FL6/IN03-04/ERP13-14/CEN07 & Valle del Ticino-Baraggia di Cameri \\
\hline 2 & UC29/M06/FL07/IN14/ERP12/CEN03 & Lagoni di Mercurago \\
\hline 3 & UC28/M04/FL08/CEN02-08 & Canneti di Dormelletto \\
\hline 4 & UC30/M05/ERP12 & Boschi di Solivo \\
\hline 5 & UC30/M05/FL16/IN01/ERP12 & Torbiera di Agrate Conturbia \\
\hline 6 & UC19/M02/FL13/IN13-17 & Alta valle del Torrente Agogna \\
\hline 7 & UC37/M11/IN17 & Monte Falò \\
\hline 8 & FL14/IN15/ERP15 & Mottarone \\
\hline 9 & U25-26/M03/FL09/CEN04-06 & Lago d'Orta \\
\hline 10 & FL9/CEN06 & Torre Buccione \\
\hline 11 & M08/FL05/IN11 & $\begin{array}{l}\text { Monte Lovagnone (settore novarese del } \\
\text { Massiccio del Monte Fenera) }\end{array}$ \\
\hline 12 & UC18/M11/FL15/IN02-03-06-10-20/ERP07-10/CEN12-14 & Fiume Sesia \\
\hline 13 & UC10-31-32/M09/FL04/IN08/ERP01 & $\begin{array}{l}\text { Piano Rosa-Bosco della Panigà-Collina } \\
\text { di Barengo }\end{array}$ \\
\hline 14 & UC5-11-15-19/M12/FL10/IN19/ERP05-06-08-9/CEN10-11-14 & Torrente Agogna (tratto planiziale) \\
\hline 15 & UC01/ERP06-07/CEN10-14 & Garzaie di Morghengo e Casaleggio \\
\hline 16 & UC17-20/FL03/IN08/ERP03-06-09/CEN09-14 & $\begin{array}{l}\text { Torrente Terdoppio-Baraggia di } \\
\text { Bellinzago }\end{array}$ \\
\hline 17 & IN02-03/ERP06-07-14/CEN13-14 & Canale Cavour \\
\hline 18 & IN02/ERP07-08/CEN14 & Roggia Biraga \\
\hline 19 & UC12/IN01/ERP11 & Palude di Casalbeltrame \\
\hline 20 & FL11/ERP08/CEN14 & Risaie tra Casalino e Granozzo \\
\hline 21 & UC09-34/M14/IN02-07/ERP06/CEN14 & Quartara-Garbagna \\
\hline 22 & UC07-20-24/IN02-09/ERP06/CEN09-14 & Risaie di Sozzago e Tornaco \\
\hline 23 & UC05/M13/FL02/IN05/ERP06-16/CEN14 & Biotopi di Borgolavezzaro \\
\hline
\end{tabular}


Tab. 2 - Elenco delle Aree protette della provincia di Novara considerate nella gap analysis. Il codice identificativo ID è lo stesso utilizzato in Figure 12.

\begin{tabular}{|c|c|}
\hline ID & DENOMINAZIONE \\
\hline EUAP0206 & Parco naturale dei Lagoni di Mercurago \\
\hline EUAP0209 & Parco naturale del Monte Fenera \\
\hline EUAP0218 & Parco naturale della Valle del Ticino \\
\hline EUAP0220 & Parco naturale delle Lame del Sesia \\
\hline EUAP0349 & Riserva naturale orientata delle Baragge \\
\hline EUAP0350 & $\begin{array}{l}\text { Riserva naturale della Palude di } \\
\text { Casalbeltrame }\end{array}$ \\
\hline EUAP0351 & $\begin{array}{l}\text { Riserva naturale speciale dei Canneti di } \\
\text { Dormelletto }\end{array}$ \\
\hline EUAP0354 & $\begin{array}{l}\text { Riserva naturale speciale del Colle della } \\
\text { Torre di Buccione }\end{array}$ \\
\hline EUAP0355 & $\begin{array}{l}\text { Riserva naturale speciale del Monte } \\
\text { Mesma }\end{array}$ \\
\hline EUAP0360 & $\begin{array}{l}\text { Riserva naturale speciale del Sacro } \\
\text { Monte di Orta }\end{array}$ \\
\hline EUAP1184 & Zona di salvaguardia del Monte Fenera \\
\hline EUAP1197 & Riserva naturale orientata Bosco Solivo \\
\hline IT1120010 & $\begin{array}{l}\text { ZPS Lame del Sesia e Isolone di } \\
\text { Oldenico }\end{array}$ \\
\hline IT1150001 & ZPS Valle del Ticino \\
\hline IT1150003 & ZPS Palude di Casalbeltrame \\
\hline IT1150004 & ZPS Canneti di Dormelletto \\
\hline IT1150010 & ZPS Garzaie novaresi \\
\hline IT1120003 & SIC Monte Fenera \\
\hline IT1120010 & $\begin{array}{l}\text { SIC Lame del Sesia e Isolone di } \\
\text { Oldenico }\end{array}$ \\
\hline IT1150001 & SIC Valle del Ticino \\
\hline IT1150002 & SIC Lagoni di Mercurago \\
\hline IT1150003 & SIC Palude di Casalbeltrame \\
\hline IT1150004 & SIC Canneti di Dormelletto \\
\hline IT1150005 & SIC Agogna Morta (Borgolavezzaro) \\
\hline IT1150007 & SIC Baraggia di Pian del Rosa \\
\hline IT1150008 & SIC Baraggia di Bellinzago \\
\hline
\end{tabular}

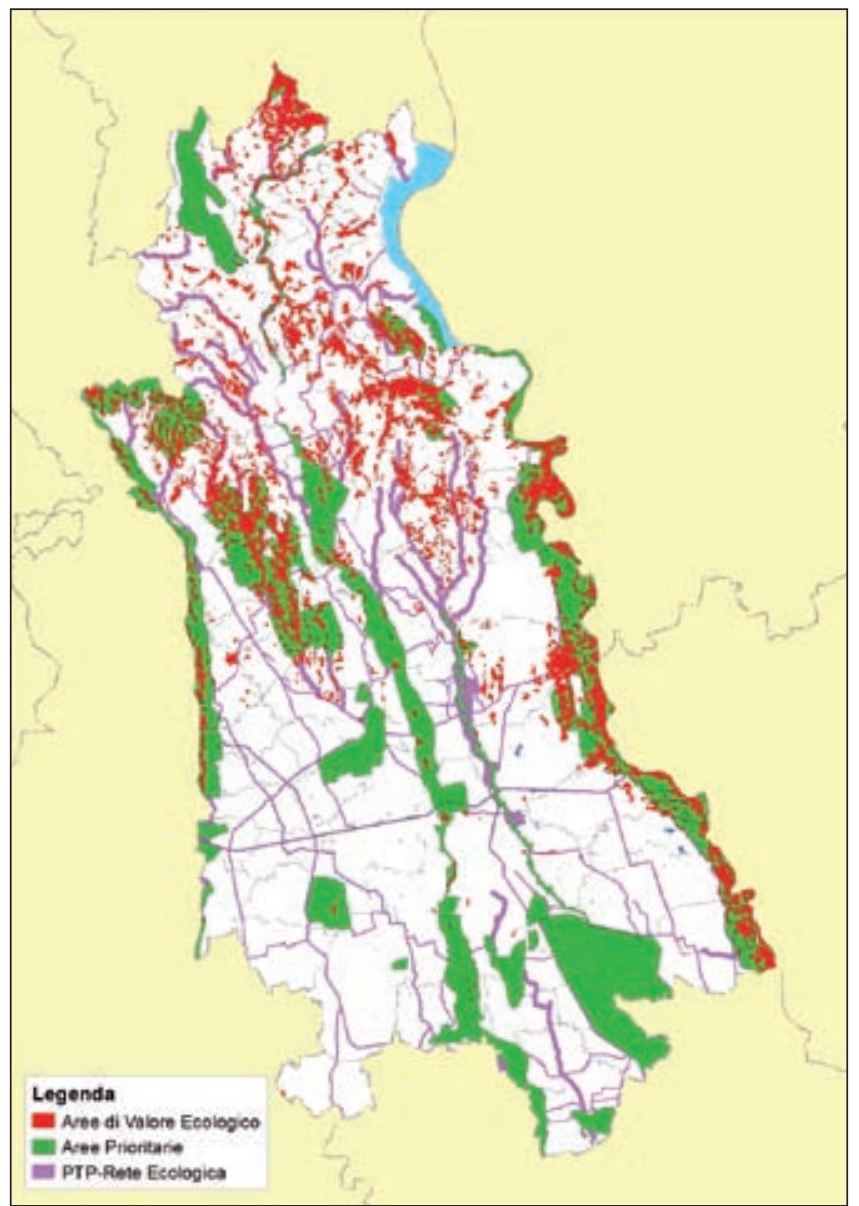

Fig. 12 - Delimitazione della Aree di Valore Ecologico definite in attraverso l'analisi GIS.

soprattutto a causa della presenza di aree boschive relativamente estese e continue che incrementano il valore modellistico. In pianura queste aree di sovrapposizione sono localizzate lungo le valli fluviali dei fiumi Ticino e Sesia e nell'area di Piano Rosa-Bosco della PanigàCollina di Barengo; nell'area collinare sono quelle di Lagoni di Mercurago, Bosco di Solivo, Torbiera di Agrate Conturbia, Monte Lovagnone. Nell'area montana sono l'Alta valle del Torrente Agogna, il Monte Falò e il Mottarone. Le aree della bassa pianura mostrano scarsa sovrapposizione per la scarsità di aree boschive. In questo caso, le valutazioni degli esperti hanno contribuito in modo sostanziale all'attribuzione di valore naturalistico ad aree caratterizzate da coltivazioni e da mosaici di coltivazioni e frammenti di aree con vegetazione naturale. Di questo occorrerà tener conto se si vorrà estendere il metodo di individuazione delle aree sorgente di biodiversità in altri territori nei quali i database dell'uso del suolo mancano di attributi relativi ad aree di importanza naturalistica non coperti da ambienti forestali.

Grazie alle nuove conoscenze acquisite è stato possibile valutare il grado di tutela formale esistente sul territorio. L'analisi delle differenze di copertura delle Aree prioritarie rispetto alle aree protette mostra come 


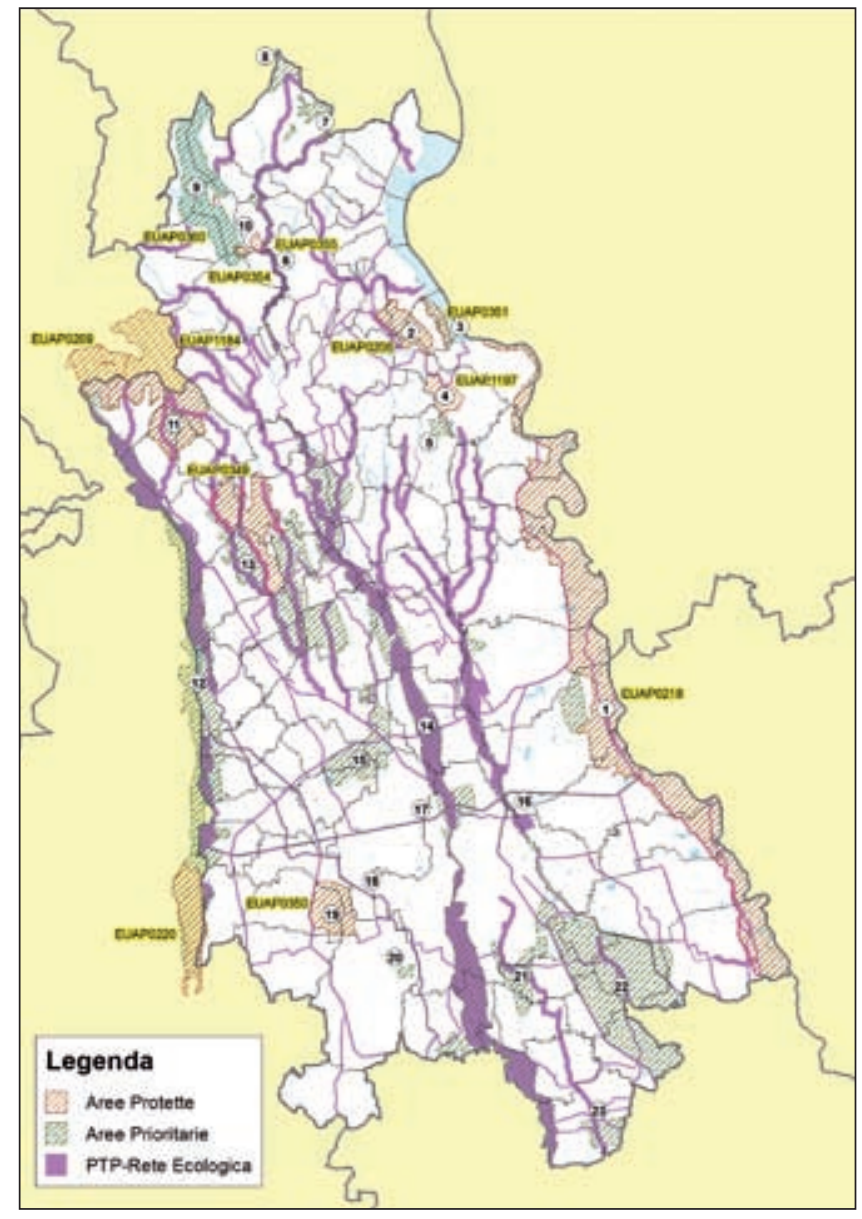

Fig. 13 - Gap-analysis, o sovrapposizione fra le Aree prioritarie per la biodiversità e le Aree protette (Parchi naturali e Riserve naturali istituiti in base alle normative regionali). La numerazione della Aree protette è la stessa della Tab. 2.

nell'area di studio tutte queste aree siano incluse nelle Aree prioritarie. Fa eccezione la Riserva Naturale del Monte Mesma, che non è stata ritenuta area importante dal numero minimo di gruppi di esperti richiesto (Fig. 14). I siti Natura 2000 (SIC e ZPS) sono tutti inclusi nelle Aree prioritarie (Figg. 14 e 15). Ciò conferma che le Aree prioritarie sono state effettivamente situate quasi totalmente, ma non esclusivamente, in aree considerate già in passato ad alto valore di biodiversità nella pianificazione delle aree protette regionali e che i siti Natura 2000 erano già stati individuati in aree confermate con la procedura qui descritta. Tuttavia, l'insieme delle Aree protette, dei SIC e delle ZPS del territorio novarese escludeva porzioni significative di territori di elevato valore per la biodiversità, ora meglio definiti con questa ricerca.

Le 23 Aree prioritarie per la biodiversità della provincia di Novara individuate attraverso la consultazione degli esperti e le aree perimetrate con l'approccio modellistico come AVE hanno fornito la base per il successivo disegno della Rete Ecologica Provinciale. Le informazioni raccolte hanno consentito di attuare la valutazione delle aree, da considerare come aree sorgente di

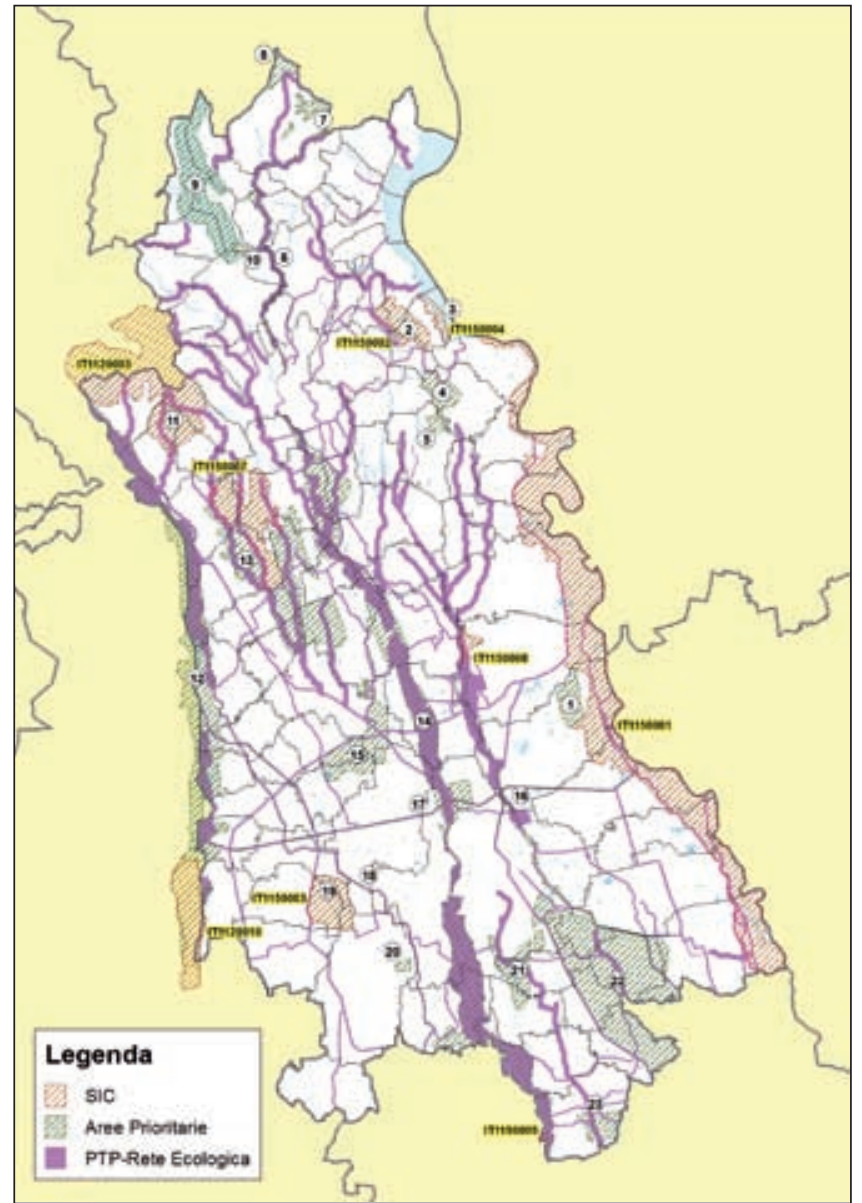

Fig. 14 - Gap-analysis, o sovrapposizione fra le Aree prioritarie per la biodiversità e i SIC - Siti di Interesse Comunitario istituiti in base alla Direttiva Habitat (Direttiva 92/43/CEE). La numerazione dei SIC è la stessa della Tab. 2.

biodiversità per le quali era importante garantire o ripristinare la connettività con le aree circostanti, attraverso l'individuazione di corridoi ecologici, di stepping stones e di aree semi-permeabili alla dispersione dei propaguli al fine di mantenere metapopolazioni vitali delle specie vegetali e animali del territorio considerato. Il successivo disegno degli elementi della rete è stato effettuato ricorrendo sia alla modellizzazione in ambiente GIS, sia ad approcci di tipo naturalistico classico. 


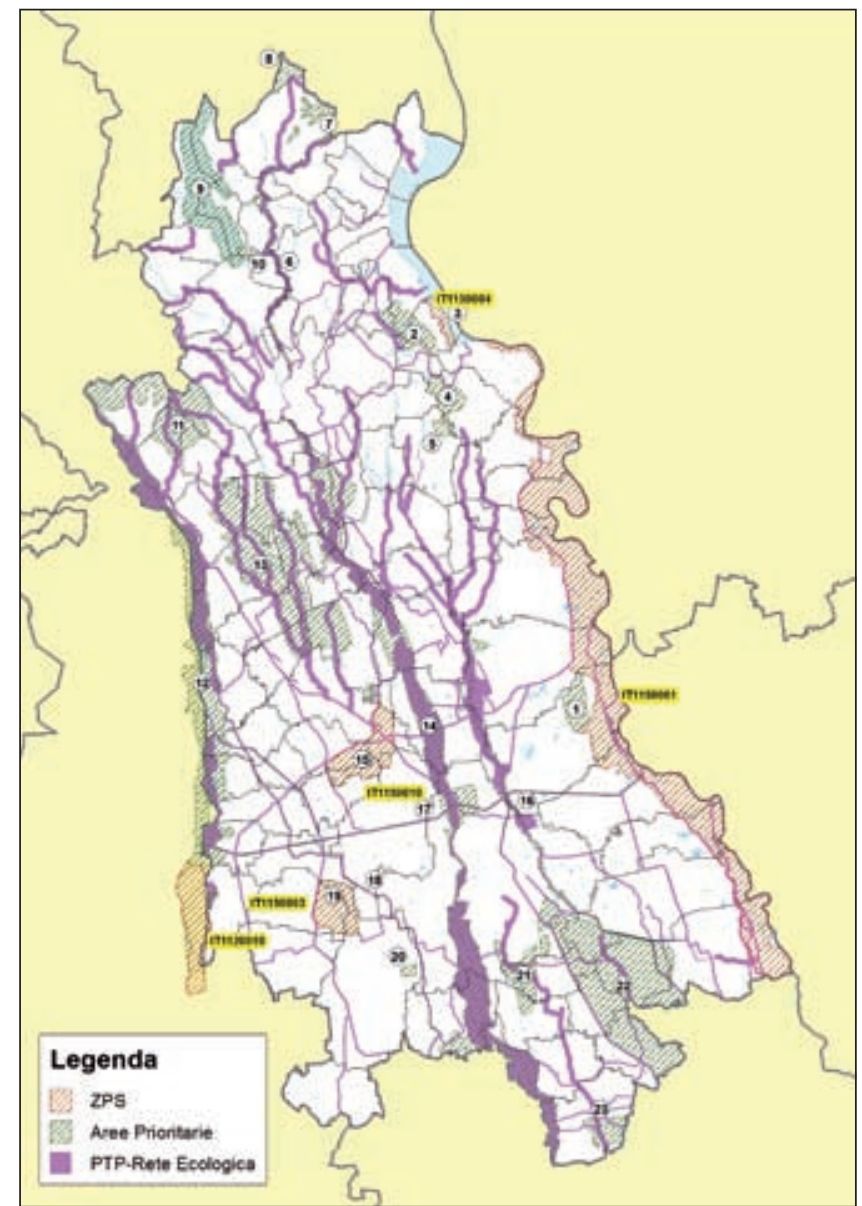

Fig. 15 - Gap-analysis, o sovrapposizione fra le Aree prioritarie per la biodiversità e le ZPS - Zone di Protezione Speciale istituite in base alla Direttiva Uccelli (Direttiva 2009/147/CE). La numerazione delle ZPS è la stessa della Tab. 2.

\section{Ringraziamenti}

Una ricerca così complessa e articolata non avrebbe potuto fornire risultati utili e in tempi così rapidi se non ci fosse stata la partecipazione competente e appassionata di numerosi esperti. Esprimiamo la nostra riconoscenza nei loro confronti per aver accettato di mettere generosamente a disposizione un sapere accumulato in anni, talvolta in decenni di lavoro sul campo e di studio e documentazione e per aver saputo interagire con altri esperti e ricercatori di discipline differenti con grande spirito di collaborazione. Per il gruppo di coordinatori di questo progetto, gli incontri di lavoro con gli esperti è stata un'occasione impagabile di arricchimento culturale. Vorremmo pertanto ringraziare tutti i 26 esperti che, insieme agli autori, hanno partecipato ai gruppi tematici: Luca Bergamaschi, Gerolamo Boffino, Angela Boggero, Mario Caccia, Mario Campanini, Pietro Cassone, Paolo Debernardi, Paolo Eusebio Bergò, Marcello Ginella, Marco Gustin, Paolo Miglio, Giambattista Mortarino, Leonardo Mostini, Raffaella Pagano, Elena Patriarca, Agostino Pela, Alessandro Re, Marco Ricci, Ettore Rigamonti, Elisa Riservato, Daniele Seglie, Alberto Selvaggi, Adriano Soldano, Giovanni Soldato, Michela Villa, Pietro Volta. Il lavoro è stato possibile grazie alla collaborazione appassionata e competente del personale della LIPU, della Regione Piemonte e della Provincia di Novara e allo studio Bertolotti di Busto Arsizio (VA). Kelsey Horvath ha gentilmente rivisto il testo dell'Abstract. Un ringraziamento va anche a Serena Arduino e a un referee anonimo per gli utili commenti e suggerimenti al testo. Il progetto è stato cofinanziato dalla Fondazione CARIPLO, Milano, nell'ambito del bando "Realizzare la connessione ecologica" del 2013.

Le mappe sono state realizzate da Nicola Gilio e da ARPA Piemonte.

Le fotografie in Appendice sono di Giuseppe Bogliani, Fabio Casale e Nicola Gilio. 


\section{PUBBLICAZIONI CITATE}

Adriaensen F., Chardon J.P., De Blust G., Swinnen E., Villalba S., Gulinck H., Matthysen E., 2003 - The application of 'least-cost' modelling as a functional landscape model. Landscape and Urban Planning, 64: 233-247.

Aimassi G. \& Reteuna D., 2007 - Uccelli nidificanti in Piemonte e Valle d'Aosta. Aggiornamento della distribuzione di 120 specie. Memorie dell'Associazione Naturalistica Piemontese. VII.

Airaudo D., Peverelli Bosser V., Fila-Mauro E., Valeria Frasca C. \& Vietti D., 2008 - Incidenti stradali con coinvolgimento di Fauna Selvatica in Piemonte. Regione Piemonte, Torino. <http://www.regione.piemonte.it/cgi-bin/agri/pubblicazioni/pub/ pubblicazione.cgi?id_pubblicazione $=1561 \&$ id_ sezione $=0>$

Alibrando M., Carrino M., Crua L., Ferrarato M., Lorusso B. \& Vietti D., 2007 - Applicazioni e modelli GIS in campo ecologico. Atti $11^{\text {a }}$ Conferenza Nazionale ASITA, Centro Congressi Lingotto, Torino 6-9 novembre 2007. <http://www.atti.asita.it/Asita2007/Pdf/262. pdf $>$

Andreone F. \& Sindaco R., 1998 - Erpetologia del Piemonte e della Valle d'Aosta. Atlante degli Anfibi e dei Rettili. Museo Regionale di Scienze Naturali Torino. Monografie, XXVI.

Arduino S., Mörschel F. \& Plutzar C., 2006 - A Biodiversity Vision for the Alps: Proceedings of the work undertaken to define a biodiversity vision for the Alps. Technical Report. WWF European Alpine Programme, Milano.

Balestrieri A., Bogliani G., Boano G., Ruiz-González A., Saino N., Costa N. \& Milanesi P., 2016 - Modelling the Distribution of Forest-Dependent Species in Human-Dominated Landscapes: Patterns for the Pine Marten in Intensively Cultivated Lowlands. PLoS ONE, 11 (7): e0158203. doi:10.1371/journal. pone. 0158203

Bari A., Converso C., Destro L., Massara M., Nappi P., Sartore L., Vietti D., Minciardi M.R. \& Rossi G., 2008 - Zone umide in Piemonte. Indicatori Ambientali. ARPA Piemonte, ISBN 978-88-7479-071-5 <http://www. arpa.piemonte.gov.it/pubblicazioni-2/pubblicazionianno-2008/zone-umide-in-piemonte.-indicatori-ambientali>

Bionda R., Mosini A., Pompilio L. \& Bogliani G., 2011 Aree prioritarie per la biodiversità nel Verbano Cusio Ossola. Società di Scienze Naturali del Verbano Cusio Ossola e LIPU - BirdLifE Italia. <http://www.scienzenaturalivco.org/Download/Relazione Aree prioritarie per la biodiversita VCO.pdf $>$

Boano G., Sindaco R., Riservato E., Fasano S. \& Barbero R., 2007 - Atlante degli Odonati del Piemonte e della Valle d'Aosta. Memorie Associazione Naturalistica Piemontese, VI.

Bogliani G., Bontardelli L., Giordano V., Lazzarini M. \& Rubolini D., 2003 - Biodiversità animale degli ambienti terrestri nei parchi del Ticino. Consorzio Parco Lombardo Valle del Ticino e Regione Lombardia. Ed. Il Guado.
Bogliani G., Agapito Ludovici A., Arduino S., Brambilla M., Casale F., Crovetto G. M., Falco R., Siccardi P. \& Trivellini G., 2007 - Aree prioritarie per la biodiversità nella Pianura padana lombarda. Fondazione Lombardia per l'Ambiente e Regione Lombardia, Milano. ISBN 978-88-8134-063-7

Boitani L., Corsi F., Falcucci A., Maiorano L., Marzetti I., Masi M., Montemaggiori A., Ottaviani D., Reggiani G. \& Rondinini C., 2002 - Rete Ecologica Nazionale. Un approccio alla conservazione dei vertebrati italiani. Università di Roma "La Sapienza", Dipartimento di Biologia Animale e dell'Uomo; Ministero dell'Ambiente, Direzione per la Conservazione della Natura; Istituto di Ecologia Applicata.

Bordignon L., 2004 - Gli Uccelli della Provincia di Novara. Provincia di Novara.

Brambilla M., Casale F., Bergero V., Crovetto G.M., Falco R., Negri I., Siccardi P. \& Bogliani G., 2009 GIS-models work well, but are not enough: Habitat preferences of Lanius collurio at multiple levels and conservation implications. Biological Conservation 142: 2033-2042.

Brunner A., Celada C., Rossi P. \& Gustin M., 2002 - Sviluppo di un sistema nazionale delle ZPS sulla base della rete delle IBA (Important Bird Areas). LIPUBirdLife Italia e Ministero dell'Ambiente, Servizio Conservazione della Natura.

Caprio E. \& Vazzola S., 2011 - I Quaderni Ambiente e Territorio. Percorsi di sostenibilità nella Provincia di Asti. Quaderno Biodiversità. Provincia di Asti.

Casale F., Rigamonti E., Ricci M., Bergamaschi L., Cennamo R., Garanzini A., Mostini L., Re A., Toninelli V. \& Fasola M., 2017 - Gli uccelli della provincia di Novara (Piemonte, Italia): distribuzione, abbondanza e stato di conservazione. Rivista Italiana di Ornitologia - Research in Ornithology, 87: 3-79.

Casale F., Sala D. \& Bellani A. (a cura di), 2014 - Il patrimonio faunistico del Parco del Ticino negli anni 2000. Parco Lombardo della Valle del Ticino e Fondazione Lombardia per l'Ambiente.

Cucco M., Levi L., Maffei G. \& Pulcher C., 1996 - Atlante degli uccelli di Piemonte e Valle d'Aosta in inverno (1986-1992). Museo Regionale Scienze Naturali di Torino, Monografie, XIX.

Dinerstein E., Powell G., Olson D., Wikramanayake E., Abell R., Loucks C., Underwood E., Allnutt T., Wettengel W., Ricketts T., Strand H., O'Connor S. \& Burgess N., 2000 - A workbook for conducting biological assessments and developing biodiversity visions for ecoregion-based conservation - part 1: terrestrial ecoregions. WWF Conservation Science Program, Washington D.C.

Ferrarato M., Vietti D., Maffiotti A. \& Sartore L., 2004 Valutazione del grado di connettività e permeabilità di un corridoio ecologico attraverso l'analisi COST DISTANCE. Atti del XIV Congresso nazionale della SItE (Società Italiana di Ecologia). Siena, 4-6 Ottobre 2004.

Fasola M., Barbieri F., Prigioni C. \& Bogliani G., 1981 Le garzaie in Italia, 1981. Avocetta, 5 (3): 107-131. 
Fortina R., Marocco R., 1994 - Distribuzione del Pelobate insubrico, Pelobates fuscus insubricus Cornalia, in Piemonte. Rivista Piemontese Storia Naturale 15: 117-126.

Framarin F., 1981 - Parchi naturali del Piemonte. Musumeci Editore, Aosta.

Framarin F., 1982 - Valutazione del sistema piemontese dei parchi e delle riserve naturali. Rivista piemontese di storia naturale 3: 5-13.

Franklin J., 2009 - Mapping species distributions. Spatial inference and prediction. Cambridge University Press, Cambridge, UK.

Furlanetto D., (a cura di) 2002a - Atlante della biodiversità nel Parco Ticino. Edizione 2002. Elenchi Sistematici (Monografie). Consorzio Parco Lombardo della Valle del Ticino.

Furlanetto D., (a cura di) 2002b - Atlante della biodiversità nel Parco Ticino. Edizione 2002. Monografie. Consorzio Parco Lombardo della Valle del Ticino.

Gruppo Di Lavoro Per La Conservazione Della Natura Della Società Botanica Italiana, 1971 - Censimento dei biotopi di rilevante interesse vegetazionale meritevoli di conservazione in Italia. Tip. succ. SaviniMercuri, Camerino.

Gruppo Di Lavoro Per La Conservazione Della Natura Della Società Botanica Italiana, 1979 - Censimento dei biotopi di rilevante interesse vegetazionale meritevoli di conservazione in Italia. Savini-Mercuri, Camerino.

Howard P.C., Viskanic P., Davenport D.R.B., Kigenyi F.W., Baltzer M., Dickinson C.J., Lwaga J.S., Matthews R.A. \& Balmford A., 1998 - Complementarity and the use of indicator groups for reserve selection in Uganda. Nature, 394: 472-475.

Knight A.T., Cowling R.M., Rouget M., Balmford A., Lombard A.T. \& Campbell B.M., 2008 - Knowing but not doing: Selecting priority conservation areas and the research-implementation gap. Conservation Biology, 22: 610-617.

Li J., Lin X., Chen A., Peterson T., Ma K., Bertzky M., Ciais P., Kapos V., Peng C. \& Poulter B., 2013 - Global Priority Conservation Areas in the Face of $21^{\text {st }}$ Century Climate Change. PLOS ONE 8: e54839.

Loyola R.D., Lemes P., Nabout J.C., Trindade-Filho J., Sagnori M.D., Dobrovolski R. \& Diniz-Filho J.A.F., 2013 - A straightforward conceptual approach for evaluating spatial conservation priorities under climate change. Biodiversity and Conservation, 22: 483-495.

Maffiotti A., Vietti D. \& Ferrarato M., 2007 - Conservation of biodiversity in the alpine lakes. Lakes management tools on a regional and local scale. Interreg IIIB Alpine Space, Progetto Alplakes. Torino. <http:// www.arpa.piemonte.it/pubblicazioni-2/pubblicazioni-anno-2007/pdf-alp-lakes-conservation-of-biodiversity>

Milanesi P., Giraudo L., Morand A. \& Bogliani G., 2015 Does habitat use and ecological niche shift over the lifespan of wild species? Patterns of the bearded vulture population in the Western Alps. Ecological Research, 31: 229-238.
Mingozzi T., Boano G. \& Pulcher C. (a cura di), 1988 Atlante degli uccelli nidificanti in Piemonte e Val d'Aosta 1980-1984. Museo Regionale di Scienze Naturali di Torino, Monografie, VIII.

Moilanen A., Leathwick J. \& Elith, J., 2008 - A method for spatial freshwater conservation prioritization. Freshwater Biology, 53: 577-592.

Patriarca E., Debernardi P. \& Toffoli R., 2012 - Piano d'azione per i chirotteri del Piemonte. Regione Piemonte. Bozza pubblicata on line: $<\mathrm{http}: / / \mathrm{www} . c e n-$ troregionalechirotteri.org/download/Piano\%20azione $\% 20$ chirotteri.pdf $>$

Pescarolo R., 1993 - I coleotteri carabidi della Baraggia di Piano Rosa (Piemonte, Novara). Rivista Piemontese di Storia Naturale, 14: 171-183.

Pescarolo R., 1996 - I coleotteri cerambicidi della Baraggia di Piano Rosa (Piemonte, Novara). Rivista Piemontese di Storia Naturale, 17: 169-174.

Riservato E., 2009 - Atlante delle libellule della provincia di Novara. Provincia di Novara, Settore Agricoltura.

Sætersdal M., Line J.M. \& Birks H.J.B., 1993 - How to maximize biological diversity in nature reserve selection: vascular plants and breeding birds in deciduous woodlands, western Norway. Biological Conservation, 66: 131-138.

Selvaggi A., Siniscalco C., Bouvet D., Antonietti A., Dellavedova R., Gallino B., Lonati M., Minuzzo C., Pascal R., Pirocchi P., Savoldelli P. \& Soldano A., 2010 - Piemonte. In: Le Aree Importanti per le Piante nelle Regioni d'Italia: il presente e il futuro della conservazione del nostro patrimonio botanico. Blasi C., Marignani M., Copiz R., Fipaldini M. \& Del Vico E. (a cura di). Progetto Artiser, Roma: 34-39.

Vietti D., Maffiotti A. \& Badino G., 2003 - Applicazione su scala regionale di un modello di idoneità ambientale per i vertebrati. Un esempio: il lupo. Atti del XIII Congresso nazionale della SItE. Como, Villa Olmo, 8-10 Settembre 2003. <http://www.ecologia.it/images/pdf/ XIII Congresso_SItE.pdf $>$

Vietti. D., Maffiotti A., Sartore L., Ferrarato M., 2004 Realizzazione del Modello ecologico BIOMOD per l'identificazione della biodisponibilità di un territorio e degli impatti previsti sulla biodiversità animale. Atti del XIV Congresso nazionale della SitE. Siena, 4-6 Ottobre 2004. 